\title{
ANÁLISIS ARQUEOLÓGICO DE PARAMENTOS. APLICACIÓN EN EL PATRIMONIO EDIFICADO SEVILLANO
}

\author{
ARCHAEOLOGICAL ANALYSIS OF PARAMENTS. APPLICATIONS \\ IN HISTORIC BUILDINGS OF SEVILLA
}

por

\author{
MIGUEL ÁNGEL TABALES RODRÍGUEZ*
}

\begin{abstract}
RESUMEN Este escrito pretende transmitir algunas nociones generales referentes a la arqueología aplicada a la edilicia histórica sevillana, y más concretamente, acerca de uno de los aspectos en los que más se ha avanzado en los últimos años: el estudio arqueológico de alzados. Dentro de este proceso, hemos desarrollado algunas líneas metodológicas específicas que han podido ser experimentadas en varios inmuebles sevillanos; seguidamente presentamos un avance sintético de nuestro modelo de investigación.
\end{abstract}

\begin{abstract}
This article pretends to present to the academical comunity the last experiences about "edilicial archaeology" in Seville. The archaeological investigations on standing structures are the result of the aplication of a metodological working line developed in last years on diferents historical buildings of our city.
\end{abstract}

\section{INTRODUCCIÓN}

En la rehabilitación de un edificio histórico el análisis arqueológico de sus paramentos se configura como una de las herramientas principales, suponiendo el montante más consistente en cuanto a información estratigráfica y estructural, superando incluso a la excavación, a los diferentes controles de obra y a los estudios multidisciplinares.

La razón fundamental estriba en la lógica de la reparación y el reaprovechamiento histórico de los elementos constructivos hasta bien entrado nuestro siglo. En la configuración de un palacio, un convento, un castillo o una iglesia sevillana se producen dos constantes seculares: la primera es el inicio de la fábrica

* Universidad de Sevilla. Escuela Superior de Arquitectos Técnicos. 
bajo un impulso general o particular, pero en la mayoría de los casos (incluso en los más importantes), reaprovechando total o parcialmente restos más antiguos a modo de cimientos. La segunda constante es el remozado continuado a lo largo de los siglos, con períodos importantes de transformación coincidentes, por lo común, con los momentos de auge de la ciudad. Así, en Sevilla, la mayor parte de edificaciones históricas parte de tres períodos cruciales en su devenir histórico: el impulso almohade (s. XII-XIII), el período de la repoblación castellana (s. XIII-XV) y el período colonial (s. XVI-XVIII). En los monumentos más antiguos es raro que cada uno de esos eventos no influya una o más veces en la construcción y se refleje en alteraciones de forjado, cambio de techumbres, apertura y cegamiento de vanos, etc...

En esos períodos de auge también es normal la transformación de los espacios y las subidas de cota, con lo cual, la estratigrafía del subsuelo tiene un papel determinante. Sin embargo, en períodos normales o de decaimiento, en los cuales no se producen grandes alteraciones y el proceso de transformación se reduce a cambios sin reflejo en el subsuelo, los alzados suelen ser testigos explícitos del cambio.

Lo que es importante para la investigación de cualquier edificio histórico, se vuelve determinante cuando ésta se inserta dentro de un proceso de rehabilitación ya que afecta en mayor grado a las decisiones de obra. En efecto, cuando se procede a la reforma de la construcción, las remociones de tierra se vinculan a una serie de operaciones que no suelen tener (o raramente) un carácter general; se trata de cambios de pavimento, que no suelen necesitar más de cuarenta centímetros de remoción para colocar mallazos, hormigón, cimiento y solería, circunscritos a algunas dependencias; también pueden abrirse nuevos sótanos puntuales (en cuyo caso sí se profundiza más); o se excavan zanjas para infraestructuras hidráulicas, etc... En definitiva, se trata de actividades que condicionan y fundamentan la investigación a nivel de excavación y que, por supuesto, inciden variablemente en los resultados finales de la obra.

Sin embargo, en la totalidad de los inmuebles, la más elemental de las operaciones vinculadas a su revitalización, pasa ineludiblemente por un picado de los muros y el enfoscado general, bien para adecentar los paramentos, para introducir regolas eléctricas o simplemente para comprobar su solidez. Como es lógico, no sólo es mayor la cantidad de información a extraer del palimpsesto, sino que de su estudio dependen decisiones relativas a la consolidación o derribo, al uso o cegamiento de accesos y vanos, a la conservación o no de pinturas murales, restos de azulejería o cubiertas, etc...

Además, su estudio agiliza la comprensión espacial y volumétrica, acercando adecuadamente la visión arqueológica a la lógica arquitectónica de la rehabilitación.

\section{ANTECEDENTES}

Pese al incuestionable valor del paramento como fuente de información arqueológica, la utilidad de los análisis de alzados se ve mermada si no se complementa con excavaciones y con otros estudios, ya que los eventos constatados en alzado se reflejan en el subsuelo mediante pavimentos asociados, cimientos reutilizados o fundacionales, rellenos, etc...y son estas realidades estratigráficas del subsuelo las que hoy por hoy suelen garantizar aspectos tan esenciales como la datación de las estructuras.

Es cierto que se han realizado esfuerzos muy importantes en el campo de la datación edilicia; destaquemos por ejemplo a Severino Fossati (1985) o Tiziano Manoni (1984) a inicios de los ochenta, datando mediante el análisis estadístico de los ladrillos italianos, o dendocronología o termoluminiscencia, etc.., pero son técnicas aún por desarrollar, con ámbitos de validez muy locales y de difícil aplicación práctica a nivel cotidiano.

Los análisis de paramentos se han incorporado recientemente a la Arqueología de la mano, sobre todo de la Arqueología Medieval y urbana. No obstante, a nivel puntual, tienen una larga tradición dentro de la arquitectura de rehabilitación, remontándose al siglo pasado; muchos arquitectos realizaron verdaderos análisis estratigráficos de los muros que iban a retocar, por lo general con fines en absoluto historiográficos, sino 
imbuidos por la lógica de la consolidación y el retoque. Destaquemos los análisis de Boni(1885) en Venecia a fines del XIX, o los planos empleados por los restauradores militares del Cuartel del Carmen de Sevilla, en 1875, en los que se diferencian cromáticamente los elementos medievales distinguidos de los contemporáneos, o los estudios de Gómez Moreno, Félix Hernández, R. Manzano o recientemente, del mismo Alfonso Jiménez (1982) en la puerta de Sevilla de Camona.

El uso de las lecturas paramentales a nivel evolutivo, como reflejo de los grandes procesos de cambio, se generalizó en la arqueología medieval británica, sobre todo en las iglesias de Rivenhall y Hadstock en Essex, estudiadas en los años setenta por Rodwell (1989). En ellos estuvo muy presente la formación teórica y metodológica de la Universidad inglesa, fruto de la cual surgieron las herramientas básicas para los futuros arqueólogos de edificios; nos referimos al método Harris, en lo referente a la documentación de estratos y los aportes sobre pluriestratificación presentados por Carver (1983). También, ya en los ochenta, se iniciaron acercamientos al análisis estratigráfico paramental como los de Hurst (1981) y Rahtz (1984). Pero sobre todo se extendió de la mano de los italianos al aplicar el método Harris a la estratigrafía muraria, destacando entre ellos Bonora (1979), Parenti (1984-1988) y Brogiolo (1988), entre otros.

La especialización progresiva de métodos y sistemas de lecturas de alzados en la arqueología del edificio ha tenido diferentes hitos. Así, en Europa Occidental, y sobre todo en Italia y España (en el resto de Europa, a menor escala), ante la proliferación de las rehabilitaciones, han surgido focos autónomos como el encabezado por Caballero Zoreda y otros investigadores castellanos, el del Servicio de Conservación de la Diputación de Barcelona (González 1985) etc... Hasta fines de la pasada década no surgieron compendios generales, fruto de las más recientes experiencias, todos ellos circunscritos al área italiana. El resto de áreas ha generado un número muy reducido de publicaciones referentes al tema, alguna de las cuales fueron emblemáticas en su momento y pueden ser consideradas pilares generales en esta disciplina: es el caso de la sistematización de Harris (1982) o de la adaptación específica de Carandini (1977). En cada zona se escribieron enfoques muy cercanos, los unos de los otros, referentes a las nuevas posibilidades del método arqueológico en la restauración, pero únicamente a fines de los ochenta y a comienzos de los años noventa se han acometido intentos formales de sistematización de la disciplina. Nos referimos a los trabajos compilatorios de Rita Tagliabue (1993).

En el resto de Europa destacaron durante los años setenta los trabajos realizados en Westfalia-Lippe y en otras zonas de Alemania, donde se estableció la figura del Investigador del Edificio (Bauforschung) como apoyo al arquitecto en el proceso rehabilitador. A nivel estratigráfico supuso un gran impulso, anterior a los trabajos de Carver Martin, el estudio sobre la problemática en los yacimientos pluriestratificados presentados por los polacos Maetzke, Rysiewska, Tabaczynski y Urbanczyk (1978).

Fue en Italia donde se produjo un mayor desarrollo en la dinámica de investigación y búsqueda de sistemas adecuados para la comprensión arqueológica de los edificios. Con la actuación en el complejo pluriestratificado de San Silvestro, en Génova, comenzó de la mano de Bonora (1979) uno de los aspectos definitorios de los posteriores estudios arqueológicos italianos: el análisis paramental estratigráfico.

En 1985, Roberto Parenti, basándose en la experiencia en la Torre A de Montarrenti, aportó el primer estudio sistemático de un edificio desde un punto de vista exclusivamente paramental, añadiendo tipologías murarias especialmente útiles para el medievo italiano.

Tras estos trabajos y otros, realizados fundamentalmente en el Norte de Italia, se multiplicaron las experiencias y aplicaciones arqueológicas, desatándose una polémica por parte de algunos arquitectos, encabezados por Renato Bonelli (1986), sobre la innecesariedad de realizar estudios arqueo-paramentales. La oposición a esta postura minoritaria la protagonizó Francovich, quien en 1988, junto con Parenti, promovió el primer compendio sobre Arqueología y Rehabilitación. En él se dieron cita los mejores exponentes de la arqueología del edificio durante la década: Manacorda, Cabona, Marino, Doglioni, Broggiolo, etc... En este sentido, Doglioni analizó el caso italiano de modo específico y propuso un sistema de estudio de los paramentos 
basados antes que en los aspectos estratigráficos y cronológicos, en las características de la fábrica y sus componentes (Doglioni 1988).

Gian Pietro Broggiolo (1988) asumió en la segunda mitad de los años ochenta un papel esencial, tras la realización de una serie de estudios destinados a la sistematización de los análisis murarios. Destaca entre todas sus aportaciones la incorporación de la jerarquización de las unidades estratigráficas según su disposición referencial, así como las gradaciones de las necesidades en los muestreos y su cumplimentación en fichas oportunas.

\section{LA REHABILITACIÓN EN SEVILLA. PRIMEROS ANÁLISIS ESTRATIGRÁFICOS DE ALZADOS}

Los estudios evolutivos de alzados se vienen realizando en Sevilla, como ya vimos en el punto previo, desde el siglo pasado; todos ellos gracias a arquitectos-arqueólogos especializados en restauración de edificios. Entre ellos, destaca desde los años setenta A. Jiménez. Desde el análisis crítico de la "Carta del Restauro" (1982), hasta su labor actual en la Catedral de Sevilla como Maestro Mayor, Jiménez ha desarrollado una extensa gama de estudios arquitectónicos e históricos plasmados en decenas de artículos y monografías, de los cuales debemos destacar, por su trascendencia y profundidad, así como por la antelación al entorno científico del momento, el trabajo realizado sobre la Puerta de Sevilla de la ciudad de Carmona, presentado en 1978. En él se propuso un profundo método "analítico" que posteriormente seguiría siendo desarrollado como proyecto docente en la Escuela de Arquitectura sevillana, y consistió entre otras bases científicas, en la restricción del ámbito descriptivo a todo aquello que pudiera ser inferido directamente de los datos sensibles, realizando su lectura a través de los atributos visuales básicos: Figura, Tamaño, Posición, Color y Textura. Las descripciones del edificio se refieren fundamentalmente, además de a las características físicas de las agrupaciones de elementos, a las características de las relaciones entre ellos, sobre todo a las de carácter tipológico y cronológico. También se hace hincapié en otros modos que se refieren a los cometidos funcionales específicos para los que fueron concebidos y a las conductas, y, por otra parte, a las instancias técnicas puestas en juego para su construcción física.

En la puerta de Sevilla, por lo que se refiere a análisis descriptivo, se atendió a las lecturas masivas, lectura espacial, superficies delimitantes, lectura funcional, tecnológica y lectura diacrónica. Además, se llevó a cabo un estudio documental amplio completado con investigaciones arqueológicas y con un extenso análisis comparativo.

Durante el año 1985, se producirá un cambio sustancial en Sevilla, con la entrada en funcionamiento del equipo arqueológico de la Delegación Provincial de Cultura de la Junta de Andalucía, a cuya cabeza operarán, primero F. Amores y luego J.M. Campos. Desde ese momento, los trabajos arqueológicos en apoyo a la restauración o rehabilitación, bien por la vía de emergencia o de urgencias, se multiplican. En todos los casos, se trata de incursiones efectuadas bajo unos presupuestos metodológicos propios de la recién creada "arqueología de gestión", imbuida sobre todo de urbanismo y evolución urbanística. Las observaciones sobre la estructura emergente son más propias de la visión de un historiador del arte que de un arqueólogo; es decir, se excava de la manera más rigurosa posible para localizar piezas que faltan en el engranaje evolutivo pero sólo complementando la información respecto a lo paramental con análisis muy generales.

No obstante, a partir de 1986, van a iniciarse dos grandes investigaciones en sendos edificios emblemáticos: en la Cartuja de Santa María de las Cuevas, F. Amores iniciará una de las mayores actuaciones jamás realizada en un conjunto monumental español, aplicando todo tipo de novedades metodológicas, destacando los estudios de alzados realizados en diferentes capillas; en los Palacios de Altamira y de Miguel de Mañara en el barrio de San Bartolomé, D. Oliva iniciará una "serie" de estudios multidisciplinares que 
desembocarían en las primeras profundizaciones específicas sobre paramentos, desde el punto de vista estratigráfico..

Desde el punto de vista de la utilización más o menos avanzada de métodos arqueológicos específicos de indagación paramental, observamos, para el conjunto de actuaciones realizadas en nuestro entorno, desde 1985 hasta 1993, los siguientes tipos-estadios de actuaciones (Tabales 1997):

1. Trabajos del tipo 1 . Se caracterizan por ser investigaciones parciales de edificios, generalmente condicionadas por su inserción a posteriori en los procesos de obra de rehabilitación, y realizadas por lo general con una visión aún muy parcial de los estudios sobre el patrimonio edificado. Comúnmente, consisten en meras excavaciones realizadas "en el edificio", pero no necesariamente con éste en el primer orden de prioridades (aunque después se intente completar la falta de atención a la estructura emergente con análisis por lo general superficiales y de carácter documental o artístico, del conjunto del monumento en cuestión). Metodológicamente son realizadas cada una según la formación del investigador y sus afinidades y generalmente no siguen el sistema de distinción de unidades estratigráficas. A este primer grupo, que coincide con nuestros primeras excavaciones en la ciudad de Sevilla pertenecen las rehabilitaciones del Noviciado de San Luis, El Convento de San Agustín, Miraflores, San Jerónimo, etc....

2. Trabajos del tipo 2. Caracterizados por su intención de análisis integral, con aspiraciones de asumir desde la óptica arqueológica el resto de los estudios multidisciplinares, generalmente bien integrados o aceptablemente insertados en el proyecto de rehabilitación. Son los estudios de la Cartuja y del Palacio de Altamira. A este grupo se pueden añadir en los años siguientes los del Palacio de Mañara, el Cuartel del Carmen y el Monasterio de San Clemente, etc...

3. Actuaciones del tipo 3. Enraizadas en el tipo 2, en cuanto a objetivos generales, se distingue de aquélla por la utilización de un sistema de trabajo autónomo y sobre todo específico, basado en las experiencias anteriores y destinado a suplir las carencias detectadas previamente, mediante la especialización del equipo de investigación básico y la definición de aquellos aspectos esenciales para la caracterización global del inmueble. Metodológicamente, como más adelante desarrollaremos, este grupo se caracterizó por su carácter experimental, poniéndose en práctica sistemas de registro diversos, indagándose en los estudios paramentales hasta cotas no superadas ni en nuestro entorno ni en el resto de Europa, etc. Pertenecen al grupo los trabajos en el Convento de Santa María de los Reyes, el Cuartel del Carmen, el Palacio de los Marqueses de Marchelina, etc...

En el Palacio de Mañara (Ojeda y otros 1990), se pone en práctica, por primera vez en Andalucía, todo un programa de estudio de alzados, desde el punto de vista estratigráfico, centrado en la estancia de las pinturas mudéjares, caracterizado por el uso rígido del sistema Harris, ya aplicado puntualmente en el Palacio de Altamira. Además se levanta con criterios arqueológicos todo el sector Suroriental de la casa, previamente a su derrumbe.

A finales de 1990, los trabajos realizados por nuestro equipo en el Cuartel del Carmen (Tabales 1990) y el Monasterio de San Clemente (Tabales 1991), ofrecieron la posibilidad de aplicar un embrionario "sistema de actuación", que se fundamentaba en la aplicación de un cuerpo de fichas de registro (unidades estratigráficas y seguimiento arqueológico); además, el estudio de los paramentos y techumbres asumía ya un papel primario y no anecdótico.

Por lo que respecta al sistema de registro, optamos en principio por seguir el modelo de fichas de unidades creado por R. Lineros para la ciudad de Carmona; así, en el Carmen 90 y en San Clemente 91. En cuanto a el resto de fichas, de seguimiento de obras y de control de bienes muebles, se utilizaron fichas propias basadas en las utilizadas en los Palacios de Altamira y Mañara, diseñadas por D. Oliva. 
El primer intento de hacer una lectura estratigráfica de alzados desde una óptica sistemática aplicando el método Harris tuvo lugar en la intervención en el Convento de Santa María de los Reyes (Tabales 1992). En nuestra tesis de Licenciatura se avanzaba una propuesta metodológica de intervención en la cual se prestaba una atención prioritaria al estudio de la "estructura aérea" (López 1994).

Tras este primer trabajo, se procedió a valorar los efectos del sistema empleado, corrigiendo y ampliando todos los aspectos metodológicos del engranaje que conformaban nuestra propuesta, de modo que, tras elegir el Cuartel del Carmen como el edificio ideal para poner en práctica lo teorizado, se pasó a aplicar de manera experimental cada uno de los elementos teóricos.

Desde la elaboración y práctica de este modelo hasta el presente, nuestro sistema se ha perfeccionado en otros edificios como el Palacio de los Marqueses de Marchelina(Tabales y otros 1995), la Iglesia de Santa Ana de Guadalcanal, la Puerta de Córdoba de Carmona (Ojeda y Tabales 1996), Catedral de Sevilla, etc...

Desde el año 1993 los trabajos relativos a las lecturas paramentales en edificios históricos sevillanos se han multiplicado considerablemente, desarrollándose experiencias novedosas entre las que destacan las dirigidas por A. Pérez Paz en iglesias como las de La Magdalena, San Andrés, etc..., así como las de R. Ojeda en la Puerta de Córdoba de Carmona (1996), etc...

\section{PROPUESTA METODOLÓGICA DE ANÁLISIS EDILICIO EN EDIFICIOS HISTÓRICOS}

En nuestra tesis doctoral (Tabales 1997), así como en otros trabajos (Tabales 1997, 3 y 4), se ha incidido sobre diferentes aspectos vinculados al proceso de intervención arqueológica en inmuebles, desarrollando el sistema metodológico diseñado para ese fin por nuestro equipo. En síntesis, el citado sistema se fundamenta sobre las siguientes pautas:

\section{ESTRATEGIA DE INTERVENCIÓN}

a- Acercamiento inicial al edificio:

- División zonal, identificación de "unidades-guía"y estancias.

- Estudio previo y pormenorizado de la estructura emergente.

- Estudio de los sistemas de adosamiento y contacto entre alineaciones.

- Análisis edilicio provisional (constatación de los tipos de muro).

- Realización de un programa de Muestreos edilicios.

- Cumplimentación de las observaciones tipológicas.

- Registros de elementos artísticos o susceptibles de conservación.

- Elaboración de una hipótesis evolutiva inicial.

b- Análisis de paramentos:

- Estudios estratigráficos.

- Análisis tipológicos.

- Constatación de los principales eventos estructurales.

c- Estudio del subsuelo:

- Realización de cortes estratigráficos.

- Apertura de cortes y zanjas-guía.

- Realización de cortes con carácter "extensivo".

d- Control de Obras:

- Control de las actividades de restauración.

- Control de las obras de rehabilitación. 


\section{REGISTRO Y MÉTODO}

a- Seguimiento del método Harris con ciertas puntualizaciones.

b- Establecimiento de unos criterios de representación gráfica esenciales, tanto en las plantas y perfiles como en los alzados de tipo estratigráfico y tipológico-estructurales.

c- Cumplimentación ordenada de registros de control:

- Unidades estratigráficas.

- Bolsas de materiales.

- Registro de cortes.

- Registro gráfico.

- Registro fotográfico.

d- Utilización de un sistema de fichas apropiado:

- Ficha de unidades única para cualquier tipo de elemento (emergente o soterrado).

- Ficha de control de obras.

- Ficha de control tipológico.

- Ficha de Muestreo edilicio.

- Ficha de estado previo.

\begin{tabular}{|l|}
\hline \multicolumn{1}{|c|}{ EQUIPO BÁSICO Y RELACIÓN INTERPROFESIONAL } \\
\hline a- Selección de los miembros esenciales del equipo y sus actividades. \\
\hline b- Los estudios multidisciplinares. Selección y valoración. \\
\hline c- La priorización de los trabajos. Condicionantes habituales. \\
\hline
\end{tabular}

Dentro del conjunto de actuaciones a realizar, el estudio de paramentos, en sus diferentes vertientes, supone la pieza fundamental, siendo además la línea de investigación en la que mayores esfuerzos metódicos hemos invertido.

Dentro del sistema, los estudios de paramentos se ejecutan en dos niveles consecutivos de actuación. En primer lugar, como parte del período inicial de auscultación de la estructura se procede a las siguientes operaciones:

- Obtención de un fichero completo en el que se reflejará el estado previo del edificio antes de la rehabilitación. Se realizará estancia por estancia, centrando nuestra atención en solerías, muros, vanos, detalles decorativos, etc..

— Identificación numérica de los ámbitos o estancias y numeración de los "Paramentos Guía", o alineaciones principales que conforman las crujías y compartimentos. Serán las primeras unidades estratigráficas de la lista que luego continuaremos. Podríamos optar por una jerarquización de las unidades tal y como propone Broggiolo (1988), pero pensamos que este tipo de disquisiciones nos alejan más que ayudarnos en el objetivo general de la comprensión global del inmueble.

- Tras esta identificación, se procede al análisis de los distintos "tipos de adosamiento"; éstos pueden ser de distinto tipo: simple, con encastres simples o complejos, coetáneos, etc..

Con ello, obtendremos una primera planta con una lectura general clara. Por lo general, el orden de los adosamientos y su tipología suelen definir con muy pocos cambios los procesos constructivos del palimpsesto. La comprensión del modo en que contactan cada una de las alineaciones principales que configuran el esqueleto del inmueble, es indispensable para empezar a tener un dictamen sistemático de su evolución. 
Para poder realizar esta lectura inicial debemos acceder a la fábrica de los muros en las zonas de unión. En el caso de que existan obras de arte, pinturas o impedimentos de cualquier tipo para poder descarnar y llaguear el muro, deberemos acudir a la excavación o a la interpretación de los contactos en la techumbre, y si esto tampoco fuera posible, recurrir en última instancia a la habitual reflexión analógica. Sin embargo, y siempre que sea posible, deben iniciarse los trabajos con el llagueado de al menos un metro cuadrado continuado, o varios puntos separados; este picado debe realizarse, por supuesto, posteriormente a un programa de catas en los enlucidos que descarte cualquier posibilidad de destrucción de pinturas o cualquier otro elemento paramental de interés artístico o arqueológico.

Posteriormente se identifican las divisiones edilicias generales; es decir, se procede a rellenar sobre una planta con las unidades paramentales guía, aquellos componentes fundamentales del muro: tapiales, ladrillo, piedra, mixtos, etc...

Con ambos estudios, el de adosamientos y esta identificación de fábricas esenciales, ya pueden establecerse las primeras hipótesis de trabajo. Éstas deberán plasmarse en una planta secuenciada en la que se otorgue a cada fase constructiva una trama distinta, acompañada de una matriz Harris interpretativa, con la secuencia cronológica provisional.

El siguiente nivel de aplicación se da en la fase de investigación propiamente dicha. En ella se procede a cumplimentar varios "ficheros de control arqueológico", destacando, a nivel paramental, el fichero tipológico y el de muestreo edilicio.

Respecto a ambos tipos de control, el uso de fichas específicas se hace imprescindible debido a varias razones. En el de Muestreos Edilicios, se ficha cada tipo distinto de aparejo, analizando a fondo sus elementos y relacionándolos estratigráficamente con otras unidades adyacentes, adjuntando número de muestra, medidas medias de los distintos elementos configurantes, esquemas de ubicación de la muestra, etc...En el Fichero Tipológico, con la intención de completar las tipologías edilicias, se fichan por tipos cada vano, arco, suelo, cimiento, aparejo, etc...enfatizando las cuestiones métricas y en general todos los paralelos y cronologías asociadas en otros edificios locales.

Seguidamente, tras la catalogación, se acometen los estudios paramentales sistemáticos. A nuestro entender existen dos vías de lectura de alzados, una con carácter estratigráfico y finalidad evolutiva derivada de los estudios arqueológicos tradicionales (medievalistas ingleses, etc...) pero sobre todo desarrollada desde la aplicación del método Harris (es la representada por Parenti 1988); y otra con carácter analítico estructural, con finalidad descriptiva, tipológica y patológica (representada por Doglioni 1988). Existe una tercera vía analítica puramente arquitectónica (Jiménez 1982), caracterizada, como ya vimos, por una minuciosa indagación física fundamentada en los aspectos tipológicos, descriptivos y espaciales.

En nuestro caso, la experiencia sevillana reciente ha ido derivando hacia la absorción de las distintas vías, vinculándolas al resto de operaciones arqueohistóricas bajo una misma óptica y finalidad, asumiendo los fundamentos evolutivos, tipológicos, estructurales, etc.., como diferentes caras de una misma moneda cuyo fin esencial es la valoración histórica de la estructura.

Por lo que respecta a los análisis de tipo "EVOLUTIVO", nuestro sistema propone el seguimiento del sistema Harris simplificado, generalizado en la medida de lo posible o circunscrito a aquellos muros que resulten básicos. Para ello, se procederá inicialmente a la citada estrategia de picado de paramentos (vide supra), llagueados o limpiezas de determinados elementos. Cada alzado será dibujado de manera individualizada a escala 1:50 preferentemente. Para identificarlo se le dotará con su número de estancia y su orientación cardinal, además de la referencia a la planta. El registro de las unidades estratigráficas se cumplimentará mediante la ficha estratigráfica diseñada para el Cuartel del Carmen (Tabales 1992), especialmente transformada para incorporar mejor los datos de alzados (Tabales 1990). 
En cuanto al modo de representar, se realizarán dibujos exactos de los contornos de las unidades principales y simplificado de las secundarias. Esto es variable según el caso, tendiéndose a la esquematización a medida que subimos la escala. Se esquematizarán los interiores de las unidades (fábricas murarias, rellenos, tapiados) salvo cuando presenten características particularmente interesantes, como en el caso de las reformas de las fábricas de muros. Cada fase, período o proceso constructivo será identificado mediante tramas. Preferimos simplificar los eventos cronológicos en un número reducido de fases mucho más comprensibles.

Las unidades principales se numerarán simplificando en un número aquellas que forman parte de un grupo homogéneo y coetáneo, como los mechinales de un forjado o las vigas de un techo. A este respecto la simplificación del método Harris, al igual que la excavación, nos permite concentrar nuestra labor de investigación en la globalidad del edificio.

Los estudios "ESTRUCTURALES" fueron incorporados a nuestro sistema de análisis en el Cuartel del Carmen y desarrollados finalmente en El Palacio de los Marqueses de Marchelina (Tabales 1995). Fruto de cotejar las experiencias propias y foráneas (sobre todo Doglioni 1988) relativas a la identificación de eventos no estratigráficos, pudimos comprobar cómo gran parte de las apreciaciones tipológicas referentes a los aparejos, o las patológicas (grietas, combamientos, rupturas estructurales o superficiales, etc...) incidían de manera providencial en la valoración arqueológica general (e incluso estratigráfica).

Ánte ésta apreciación optamos por crear un modelo propio de indagación en ese campo, tradicionalmente vetado a los arqueólogos, materializándose en varias tipologías fruto de la comprobación científica durante la última década en excavaciones urbanas en la ciudad de Sevilla. Ni que decir tiene que se trata de un modelo embriónico que tenderá a ser transformado y mejorado a medida que se vayan incorporando nuevos datos.

Se dividen en dos:

1- Estructurales: Estableciendo mediante una simbología desarrollada por M.A. Tabales, (pero basada en Doglioni 1988) las relaciones físicas más importantes (rupturas, erosiones, adosamientos, grietas, etc...).

2- Tipológicos: Mediante un código creado al efecto, se identifican: aparejos, vanos, añadidos y enlucidos.

En el caso del aparejo, se subdivide en 4: el ladrillo (TIPO I); con una subdivisión en tipos basada en Clairac y Parenti, añadiendo tipos sevillanos identificados hasta el momento. La Piedra (TIPO II); siguiendo a Parenti, complementado por M.A. Tabales. Los Mixtos (TIPO III); siguiendo a Parenti, complementado igualmente. El Tapial (TIPO IV); 4 subtipos (de momento) identificados en Sevilla en función de sus características formales. A este respecto, la experiencia aún es insuficiente como para valorar mejor los aspectos derivados de la composición de los cajones (aunque estamos en ello), mientras que los aspectos métricos atraviesan por una fase de indagación que posiblemente desemboque en nuevas tipologías en breve.

En el caso de los vanos, hemos establecido una clasificación basada en nuestra experiencia en Sevilla. al igual que en los enlucidos.

\section{RESUMEN}

Si bien se observan antecedentes en otras épocas, la indagación con fines arqueológicos y metodología específica sobre la estructura emergente nace, tal y como hoy la entendemos, en Italia, a fines de los setenta, como fruto de un interesante momento científico que recoge la tradición medievalista aplicada a las excavaciones urbanas en toda Europa. Todo ello unido a un proceso generalizado de rehabilitación de edificios históricos, desemboca en un desarrollo acelerado de esta disciplina en las dos últimas décadas.

En España, casi coetáneamente surgen estudios metodológicos y aplicaciones específicas, sobre todo en Cataluña y Castilla, pero generalmente circunscritas a pequeños inmuebles sin demasiada complejidad 
evolutiva, lo cual incide negativamente en la necesidad de perfeccionar los sistemas de lectura de alzados, salvo a nivel gráfico donde se agilizan los sistemas de representación gracias a la fotogrametría.

Por lo que respecta a Sevilla, es a mediados de los ochenta cuando se produce el proceso dinamizador que desembocará en nuestra propuesta metodológica de análisis paramental. Podemos encontrar antecedentes en otras épocas, pero a nivel estratigráfico y vinculado al reciente período de obras vivido por nuestra ciudad, se aprecian varios hitos fundamentales. Puede considerarse la investigación en la Cartuja de Sta María de las Cuevas como el punto de partida, complementado con los trabajos en los palacios de Altamira y Mañara. Por nuestra parte, el sistema propuesto se fundamenta y consolida en las intervenciones en los Monasterios de San Clemente, El Carmen, Santa María de los Reyes, los palacios de los Marqueses de Marchelina, San Leandro, etc...

Este sistema consiste, en lo que respecta al estudio de lo emergente, en la ejecución de varias fases de indagación: una primera auscultación en la que se identifican los procesos básicos y se establece una primera hipótesis, a la par que se formalizan distintos controles tipológicos y de muestreo paramental; $y$ una segunda fase en la que se investigan los alzados desde varias ópticas (la evolutiva, la estructural y la tipológica).

El resultado, imprescindiblemente unido al resto de estudios arqueológicos (subsuelo, controles de obra, artísticos, documentales y otros análisis multidisciplinares) se plasmará en la interpretación diacrónica y procesual definitiva.

\section{BIBLIOGRAFÍA}

BONELLI, R. (1986): “Archeologia stratificata e Storia dell'Architettura" en Architettura. Storia $e$ documenti, 2.

BONI, G. (1985): Il muro di fondazione del Campanile di San Marco a Venezia en Archivo Veneto, XXIX,2. BONORA, F. (1979): "Nota su un archeologia dell'edilizia" en Archeologia Medievale, VI, Firenze.

BROGGIOLO, G.P. (1987): Apunti ed imagini per l'analisi stratigrafica dell'edilizia storica. (1988): Archeologia dell'edilizia storica, Como.

CARANDINI, A. (1977):"Per una carta dello scavo archeologico" en Archeologia Medievale VI, Firenze.

CARVER, (1983): "Valutacione, strategia ed analisi nei siti pluristartificati" en Archeologia Medievale X, Firenze.

DOGLIONI, F. (1988): "La ricerca sulle strutture edilizie tra aecheologia stratigrafica e restauro architettonico". Archeologia e Restauro dei Monumenti. Firenze.

FOSSATI, S. (1985): "La datazione dei matoni: una proposta di metodo" en Archeologia Medievale XII, Firenze.

GONZÁLEZ, A. (1985):Recerca i disseny. El monument com a document historic i com objecte arquitectonic viu. Barcelona 1985.

HARRIS, C. (1991) Principios de estratigrafía arqueológica. Barcelona. (traducción del original inglés, Londres 1979).

HURST, H. (1981): “La stratigarfia degli elevati” en Atti del Convegno Come L'archeologo opera sul campo, Siena.

JIMÉNEZ, A. (1982): “Carta del Restauro,72”. Colegio Oficial de Arquitectos de Andalucía Occidental. Sevilla.

(1982): "Notas para una teoría integrada de la restauración de monumentos" en Cuadernos de Construcción No 2. Mayo 1982.

MAETZKE, G., RYSIEWSKA, T., TABACZYNSKI, S., URBANCZYK, P. (1978): "Problemi dell' analisi descrittiva nelle ricerche sui sitti archeologici pluristratificati" en Archeologia Medievale IV, Firenze.

MANNONI, T.(1984) "Metodi di datazioni dell'edilizia storica" en Archeologia Medievale XI, Firenze. 
OJEDA, R. TABALES, M.A. (1994): "Estudio diacrónico de la ocupación del edificio islámico ubicado bajo la Casa de Mañára (Sevilla), tres usos, tres culturas (ss. XII-XV)." Actas del IV Congreso de Arqueología Medieval Española, Alicante.

- (1996): "La investigación arqueológica en Bienes Inmuebles. Metodología aplicada en la Puerta de Córdoba de Carmona", en Boletín del IAPH $n^{\circ} 15$, Sevilla, Junio de 1996.

OJEDA, R.; OLIVA, V. y TABALES, M.A. (1990): "Intervención arqueológica de apoyo a la restauración del Palacio de Mañara, Sevilla. A.A.A. Sevilla.

PARENTI, R. (1984): "Le strutture murarie: cenni sul metodo di analisi e prime considerazioni" en la comunicación "Scarlino, un castello della costa toscana tra storia e archeologia", in Castelli, storia e archeologia (a cura di R. Comba e A. Settia), relazioni del Convegno tenuto a Cuneo il 6-8 diciembre 1981, Torino.

- (1985): "La torre A: una lettura stratigrafica" en la comunicación "Il progetto Montarrenti (Siena), Relazioni preliminare 1984" Archeologia medievale, XII, Firenze.

- (1985):"La lettura stratigrafica delle murature in contesti archeologici e di restauro architettonico", Restauro e città, 2.

_ (1985): "I mareriali e le tecniche construttive, Un villaggio di minatori e fonditori di metallo nella Toscana del medioevo: San Silvestro (Campiglia marittima), Archeologia medievale, XII. Firenze.

_ (1986): "La torre B, in il Proggeto Montarrenti (Siena). Relazioni preliminare, 1985" Archeologia Medievale, XIII. Firenze.

- (1987): "Una proposta di classificazione tipologica delle murature postclassiche", in Conoscenze e Sviluppi teorici per la conservazione di sistemi construttivi tradizionali in muratura, Padova.

— (1988): "La techniche di documentazione per una lettura strattigrafica dell'elevato" Archeologia e restauro dei monumenti, (A cura di Ricardo Francovich). Firenze.

(1988): "Alcune osservazioni sulla prassi operativa nella lettura stratigrafica dell'edilizia" Notiziario di archeologia medievale, 48, Genova.

(1988):"Sulle possibilità di datazione e classificazione delle murature" Archeologia e restauro dei monumenti. Firenze.

RODWELL, W. (1989): Church Archaeology. London.

RAHTZ,P.H. (1984): "Archaeologists and architects: Areas of communication, collaboration and conflict" en Archaeological papers from York. York.

TABALES, M.A. (1990): "Intervención arqueológica de apoyo a la restauración del Cuartel del Carmen, Sevilla”. A.A.A. Sevilla.

(1991): "Intervención arqueológica en el Real Monasterio de San Clemente, Sevilla". A.A.A. Sevilla. (1992): Intervención arqueológica en el Convento de Santa María de los Reyes de Sevilla, A.A.A. Sevilla.

(1992): "La casa mudéjar", en “La Casa-Palacio de Don Miguel de Mañara, Sevilla.

(1992): Metodología arqueológica aplicada a la Rehabilitación de edificios. El Convento de Santa

María de los Reyes de Sevilla. Tesis de Licenciatura, Universidad de Sevilla, Dpto. de Prehistoria y Arqueología, (inédito). Sevilla

(1995): "El edificio musulmán excavado bajo el Convento de San Clemente de Sevilla". Catálogo exposición "El último siglo de la Sevilla islámica", dirigida por la Dra. Magdalena Valor Piechotta. Sevilla. (1997): Intervención arqueológica en el Real Monasterio de San Clemente. Sevilla.

(1997): "Los cimientos de la Giralda", en Aula Hernán Ruiz II, Vademecum, Sevilla.

(1997): "Arqueología y Rehabilitación de edificios históricos. Propuesta de intervención y análisis global a través de la experiencia sevillana" en Boletín del IAPH, n²0, Sevilla 1997.

TAGLIABUE, R. (1993): Architetto e Archeologo. Confronto fra campi disciplinari. Milano. 

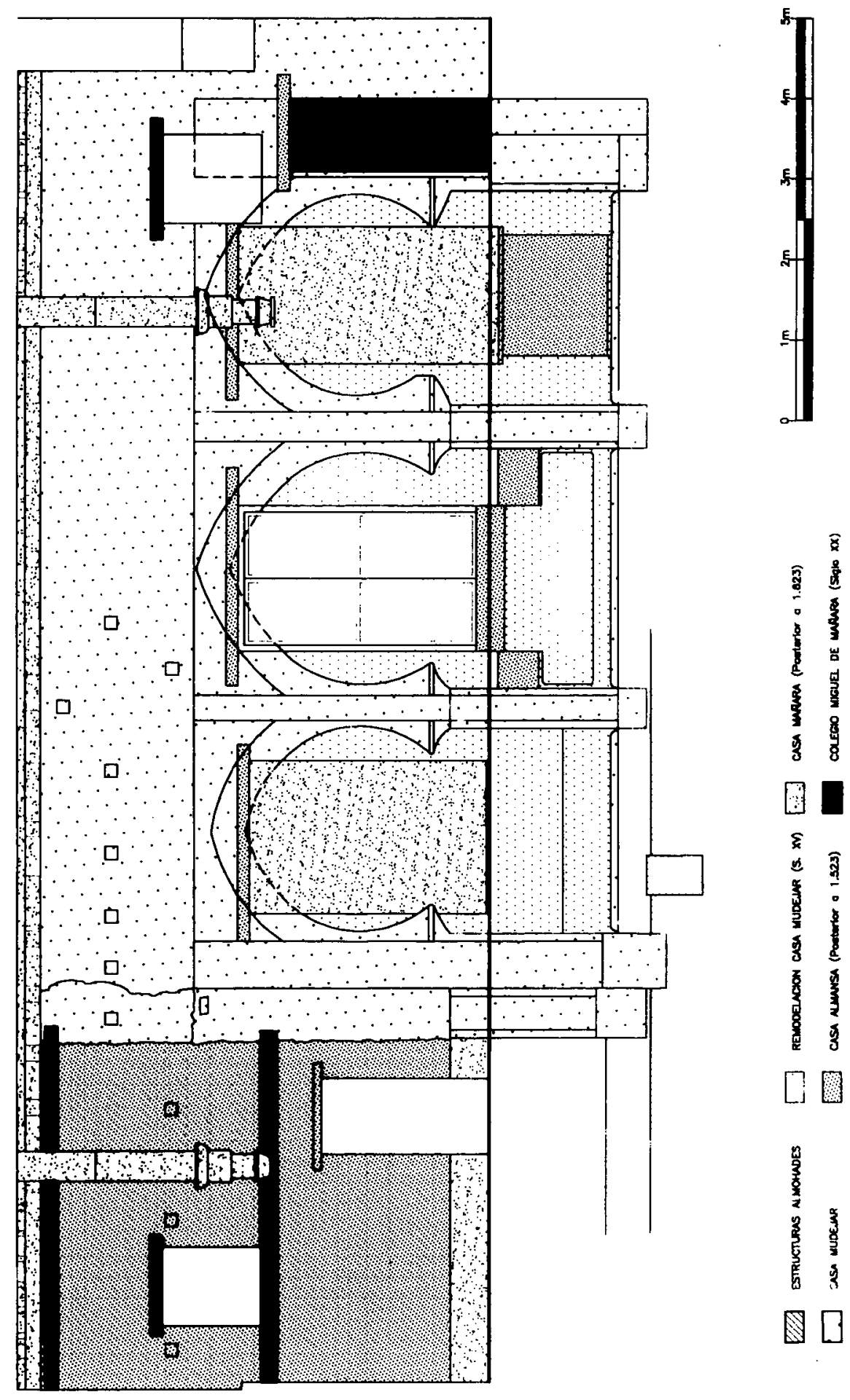

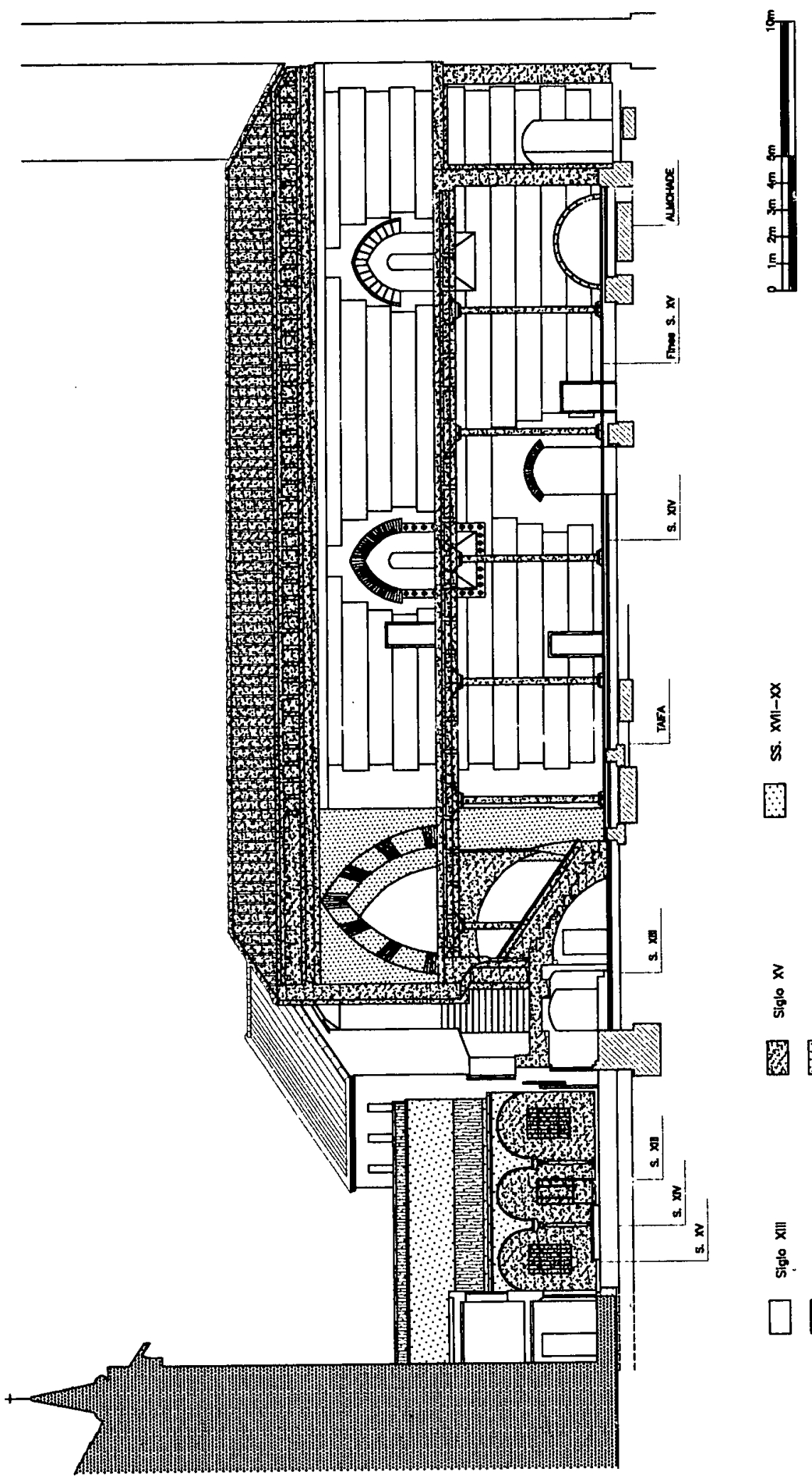

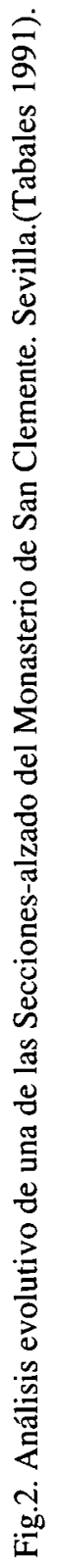

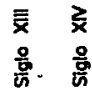

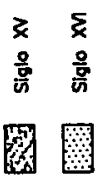

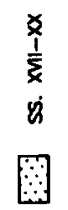




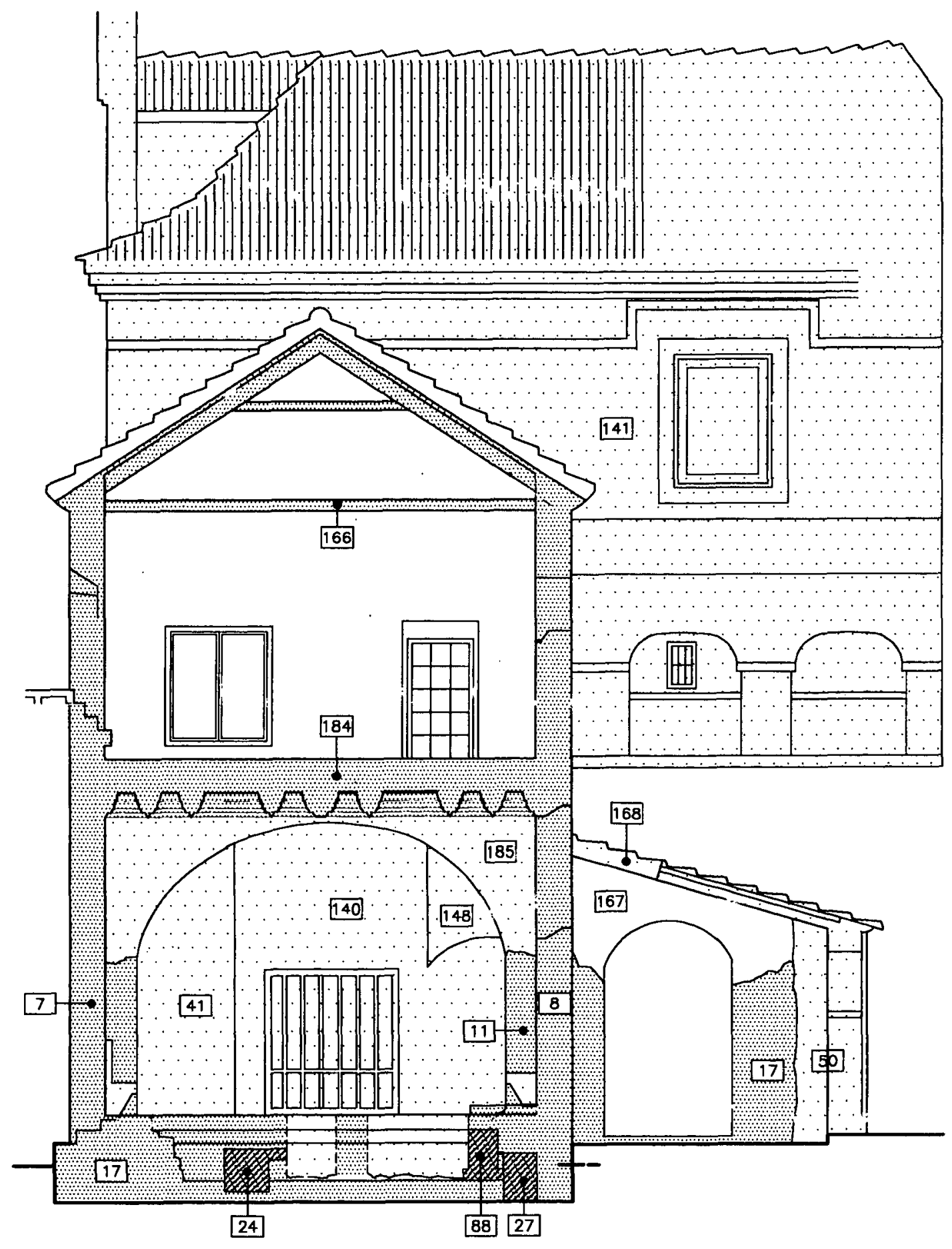

Inicios del S. XVI

Mediodos del S: XVIII

Fig.3. Análisis evolutivo de los alzados de la antigua iglesia descubierta en el Convento de Santa María de los Reyes. Sevilla. (Tabales 1992). Primeras aplicaciones del sistema Harris en Sevilla. 


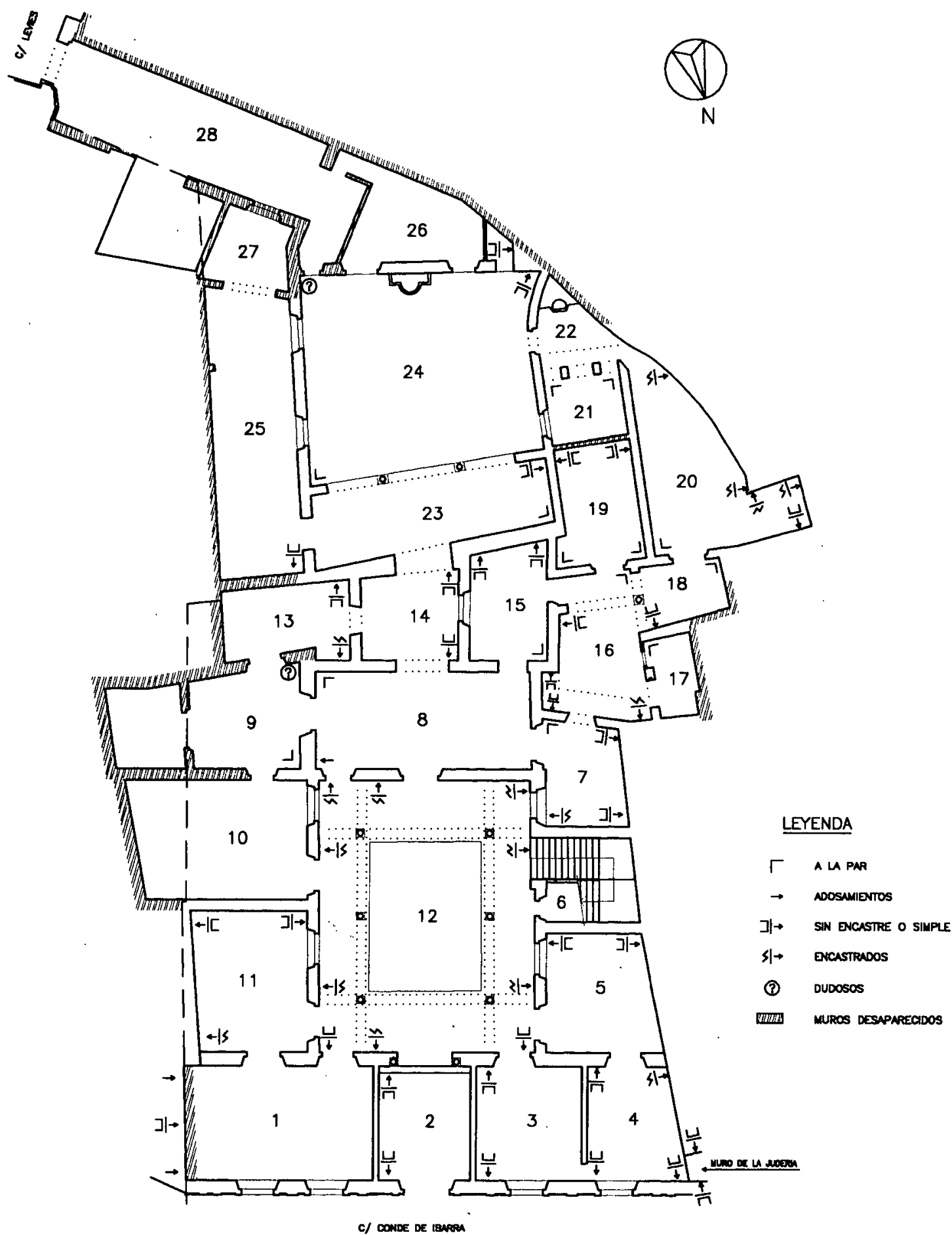

Fig. 4. Sistemas de adosamiento. Palacio de los Marqueses de Marchelina (Tabales, Pozo, Oliva 1994). 

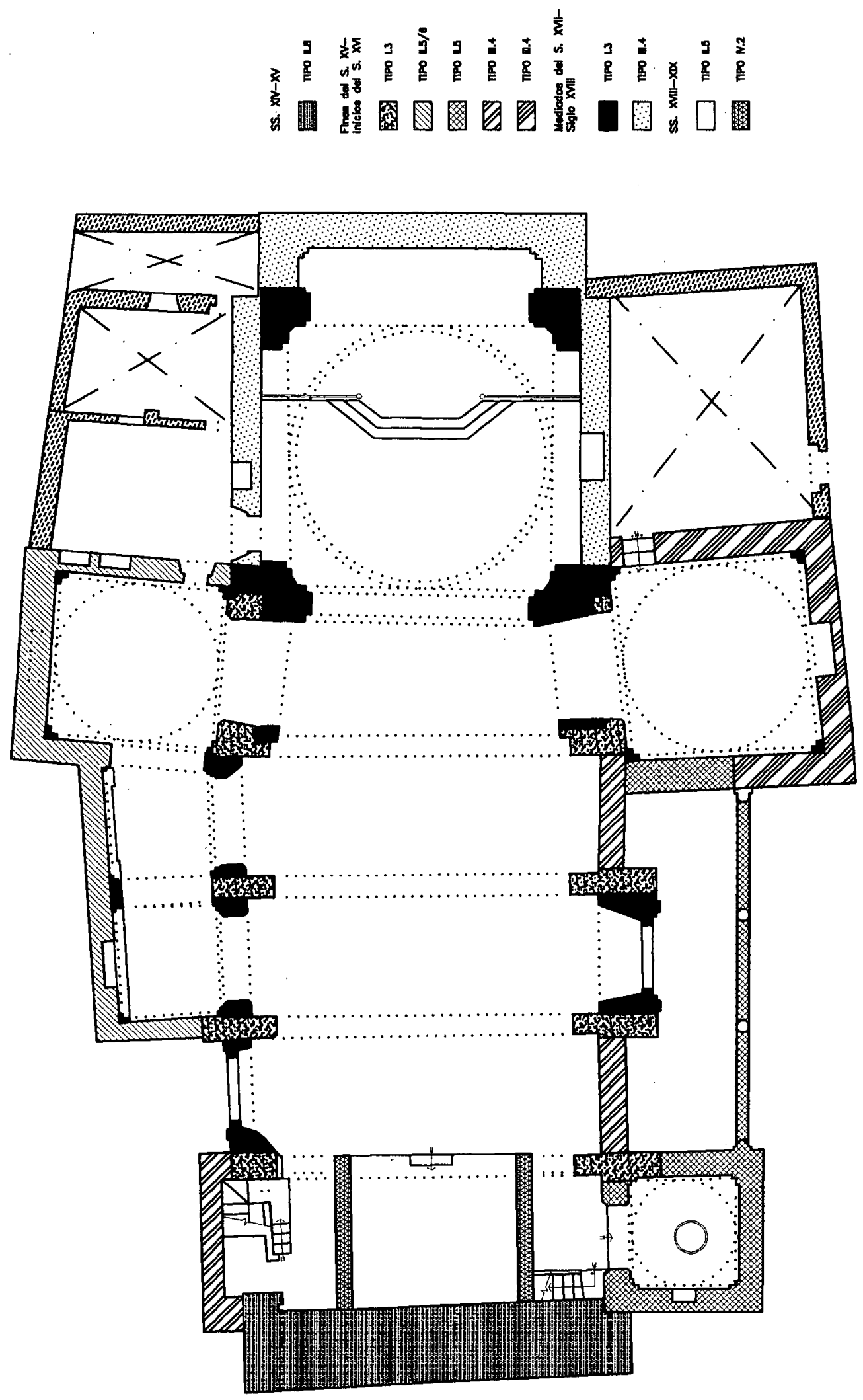

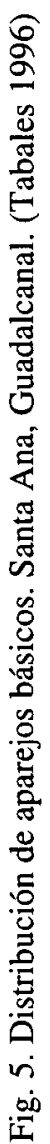




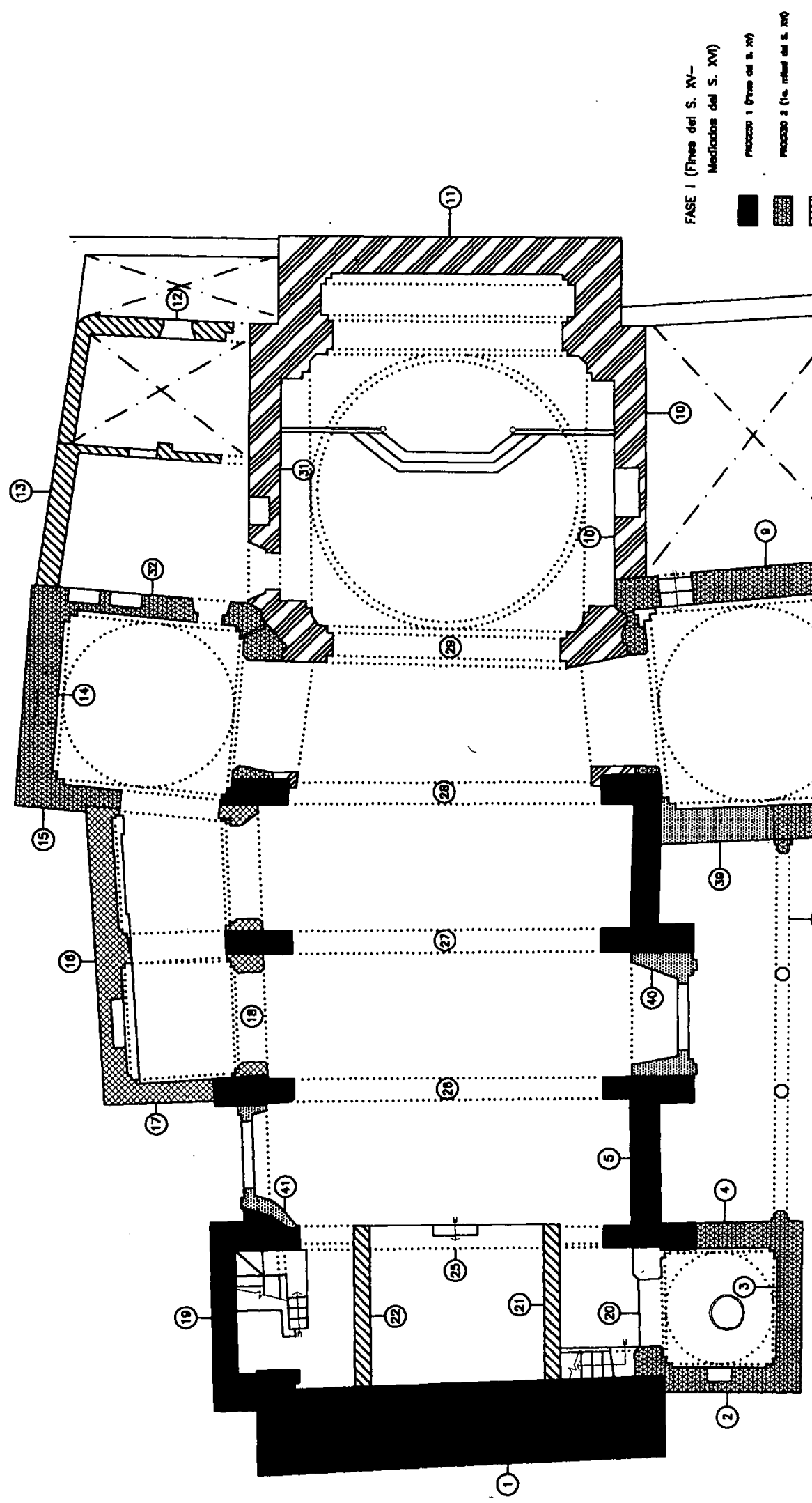

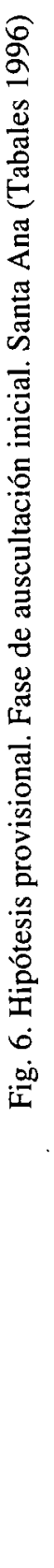

ISSN: 1133-4525 ISSN-e: 2255-3924

SPAL 6 (1997)

http://dx.doi.org/10.12795/spal.1997.i6.15 


\section{PROPUESTA DE SIMBOLOGIA PARAMENTAL}

SIMBOLOS PARAMENTALES (RELACIONES ENTRE UNIDADES Y CARACTERES).

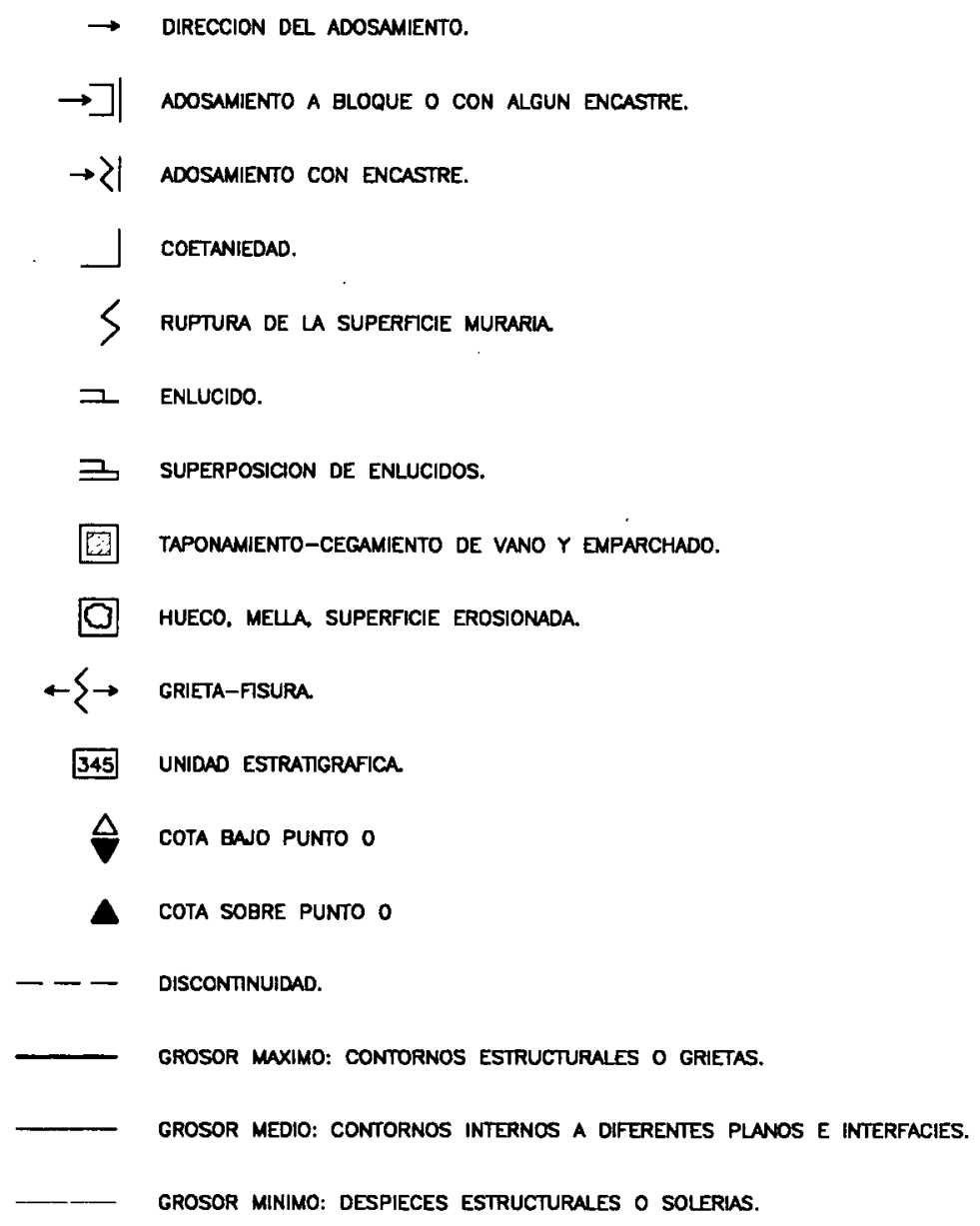

SIMBOLOS TIPOLOGICOS

(12) TPO DE APARENO.

1 TPO DE VANO.

(3) TPO DE ENLUCIDO.

(3) Elementos ARMDIDOS.

Fig.7. Propuesta de simbología paramental.(Tabales 1994). 


\section{A. TIPO DE APAREJOS}

I. APAREJO DE LADRILLO

$$
\begin{aligned}
& \text { Se utilizaro la clasificación de Clairac- } 1.877 \text { completada con la de Parenti-1.988, numerada } \\
& \text { del } 1 \text { al } 14-\text { MATR-1.993. }
\end{aligned}
$$

\section{I.1 A SOGA (PER FASCIA)}
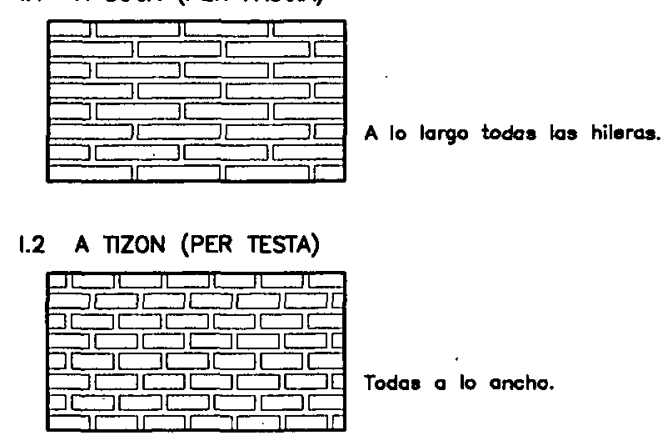

1.3 A SOGA Y TIZON (INGLES, A BLOQUE)

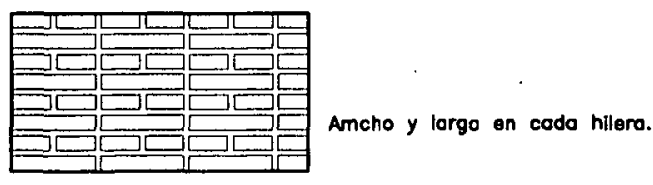

I.4 INGLES ANTIGUO (PERGIARDINO)

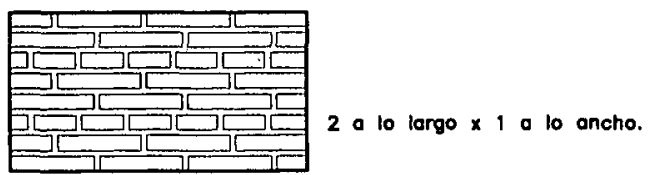

1.5 BELGA (INGLES A CRUZ)

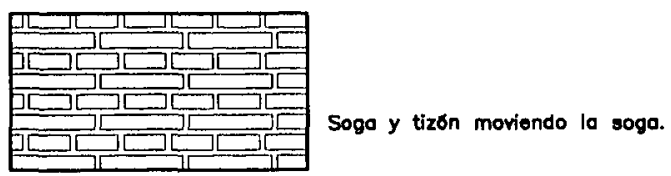

1.6 FLAMENCO (GOTICO O DLATONICO)

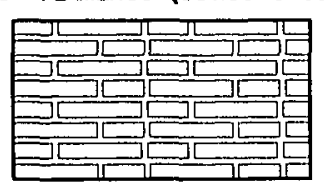

Sogo y tizón en codo hiloda.

\subsection{HOLANDES}

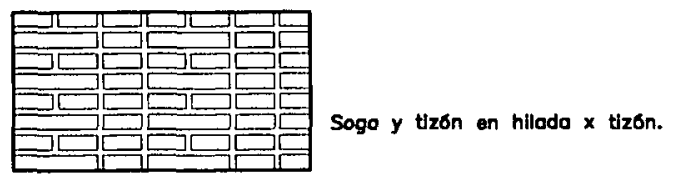

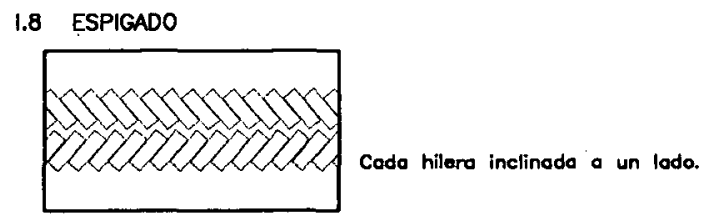

1.9 EMPLECTON

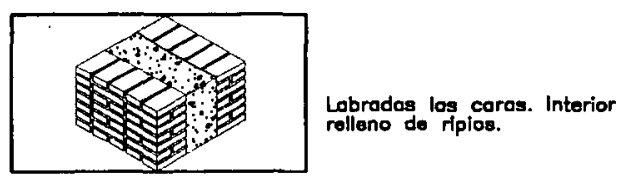

I.10 PER COLTELLO (A CUCHILO)

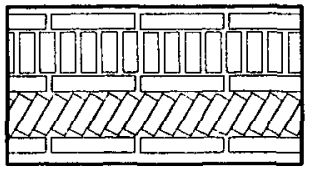

Atternondo de conto y algunos inclinodas.

I.11 PER COSTA (IN FOGUO)

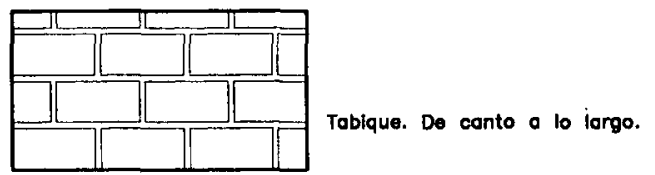

I.12 RAT TAP

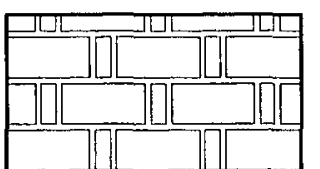

Altemando en cada hilero de conto y de lango.
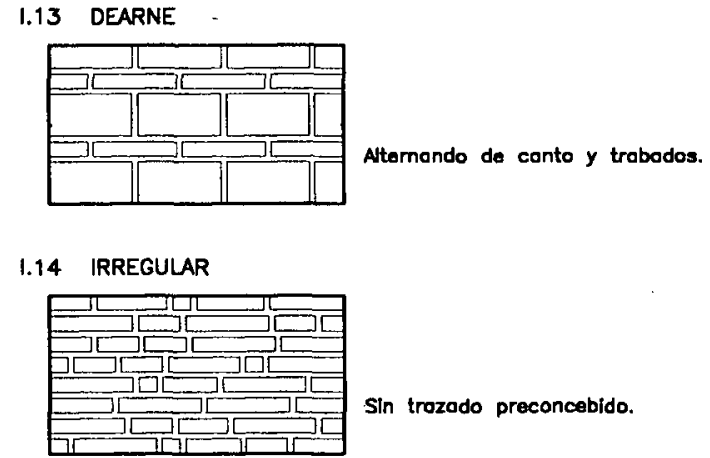

Fig.8. Tipos de aparejo. Ladrillo. (Clairac, Parenti) 
So utilizoro la clanificocion de Parenti-1.988. Tras el numero II, la tipología del 1 el 16 mos los nomeros 17 (romono) y 18 (cklopeo) de Clairoc-1.877.

II. Aineodo subhorizontal irregulor.

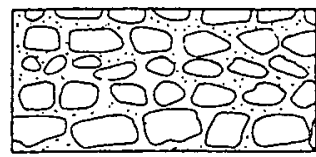

II.2 Alineado subhorizontol boslo con sillorejo y ladrillo.

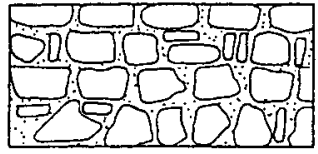

11.3 Argamasc con agregados de guijarras a cerómica.

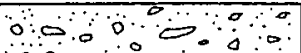

$0000 \div 0 \div$

$\because \because \because \div 00$

$\because 0 ; 0 ; 000$

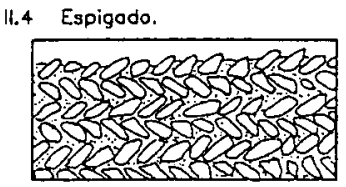

1..5 Irregular $\sin$ línea con bloques espociados, con 0 sin relleno de esquirlos.

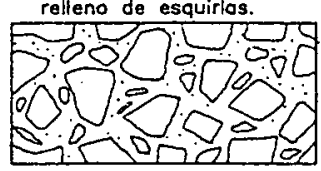

II. 6 Irregular con bloques alorgados.

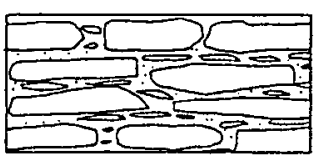

il.7 Irregular con sillarejo o mampuesto espaciodo.

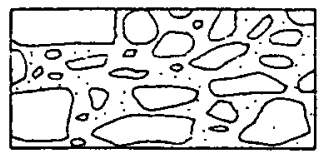

II.B Alineados subhorizontales y porolelos con sillarejo.

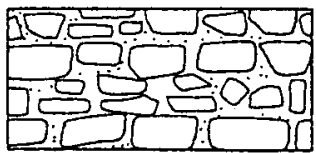

11.9 Sin alinecmiento con sillares, mampuesto o sillarejo con rollenos de ladrillo.

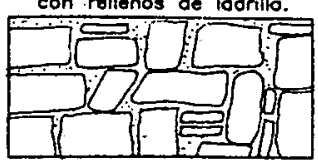

110 Alineado horizontal y paralelo con bloques de

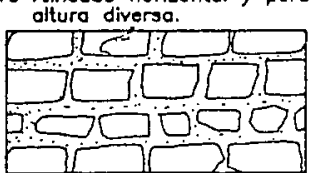

11.11 Alineodo horizontal y paralelo con bloques

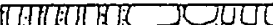

U:Uu

$300=0$

1000000000000

11.12 Alineados horizontoles y porolelos con sillores encuodrodos.

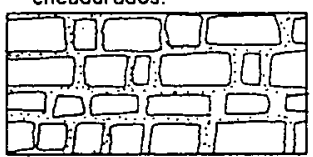

1113 Alineados ondulados con a sin esquirlos.

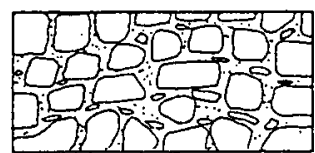

II.14 Alinodos paralelos y horizontales tipo orabe.

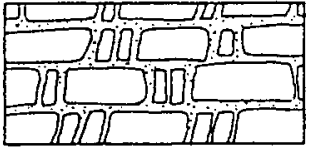

II. 15 Pseudoisodomo.

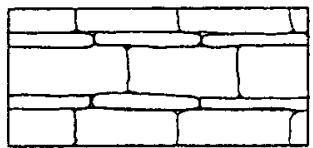

II. 16 isodomo.

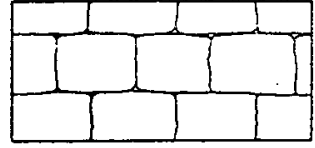

11.17 Romano o "ontiguo".

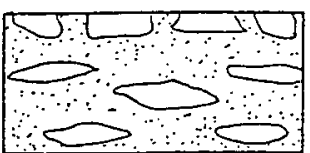

II.18 ciclopeo.

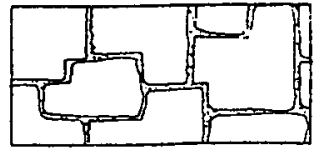

Fig. 9. Tipos de aparejo. Piedra.(Parenti) 
III. APAREJO MIXTO (Piedro y ladrillo).

So seguiro to clasificoción de Porenti-1.988 adembs to de MMTR-1.994 del 5 al 9.

Iil.1 Alineamiento subhorizontal con lodrillos o piedros $y$ cuños.

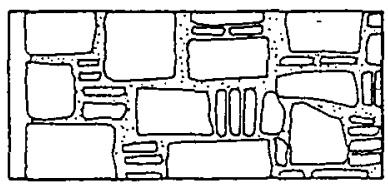

111.2 Linecs de piedra seporando ladrillo.

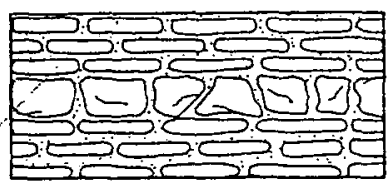

III.3 A coseto con "pillori".

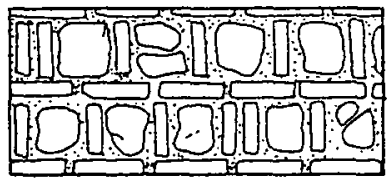

III. 4 Líneos de lodrillo seporando mampuesto.

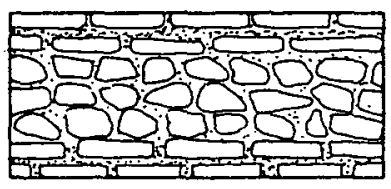

Ill.6 Lodrillo irregulor y sillares.

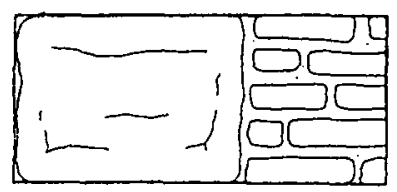

III.7 Lodrillo seporando sillores (pseudoisodomo mixto)

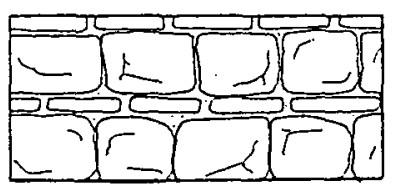

III.8 Mixto irregulor (ladrillo, sillorejo, mampuesto).

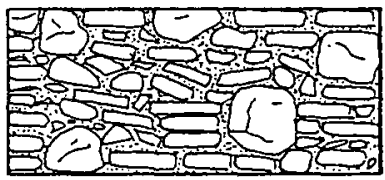

III.9 Mixto olternondo hilados oblicuas (ladrillo, piedro) o verticales

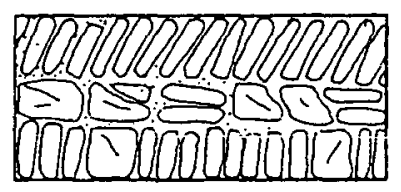

Ill.5 Líneas de ladrillo y sillorejo irregular.

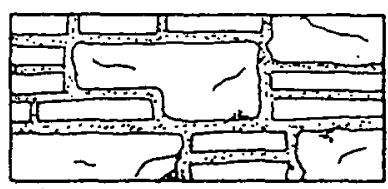

Fig.10. Tipos de aparejo. Mixtos. (Parenti, Tabales) 
IV. APAREJO DE TAPIAL.

Se seguirt lo closificocion de MATR-1.994, inicialmente del 1 al 12 .

IV.1 Tierro compocto por uno o mas hiladas de ladrillo y cal vertical. Z6calo de ladrillo.

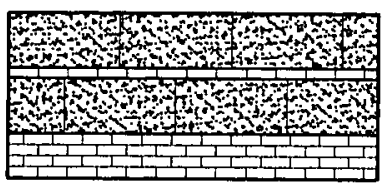

N.2 Tierra poco compocto separado por hiladas de ladrillo y cal vertical. zocalo de ladrillo.

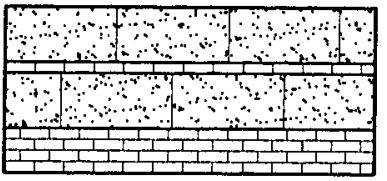

IV.3 Argamaso en general con cimentoción a zócolo de ladrillo y separación de cal.

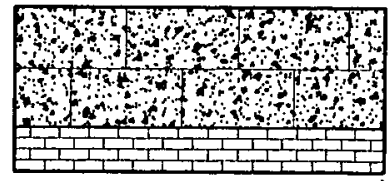

N.4 Argamasa con cimentación de mampostería y seporación de col.

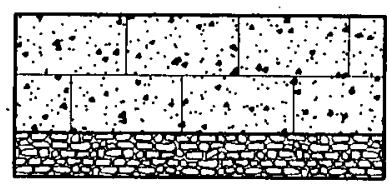

N.5 Tierro compocto con cimentación o z6colo de lodrillo y separación de cal.

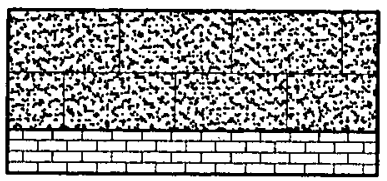

N.6 Tierro poco compacta con cimentación o z6calo de lodrillo y separación de col.

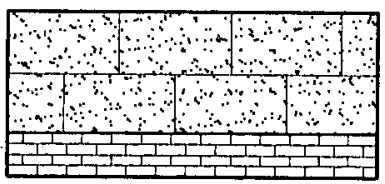

N.7 Tierra compacto con cimentacion de mompostería y separación de cal.

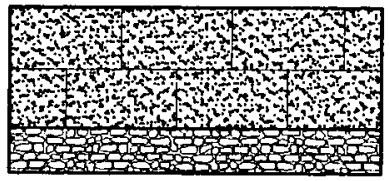

IV.8 Tierra poco compacto con cimentocion de mampostería y seporación de cal.

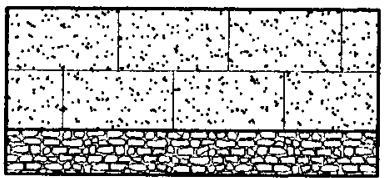

N.9 Cimiento de ladrillo "mudejar" con tapiol.

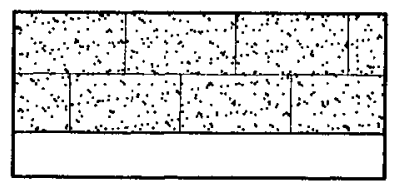

N.10 Argamoso compacto con obundantes guijarros, con separación de cal sin zapata.

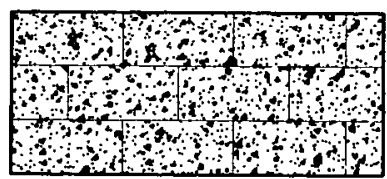

N.11 Argamoso compacto con poco guijarro, separación de cal $\sin$ zapata.

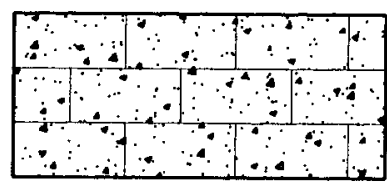

N.12 Tierro prensada con cal, ain zapato ni ladrillo.

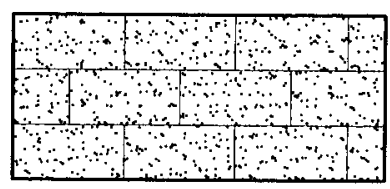

Fig. 11. Tipos de aparejo. Tapial. (Tabales 1994) 
B. TIPOS DE VANOS (más frecuentes en la edilicia sevillano).

1. ADINTELADO CON COSTERO

Genoralmente Inserto o posterior.

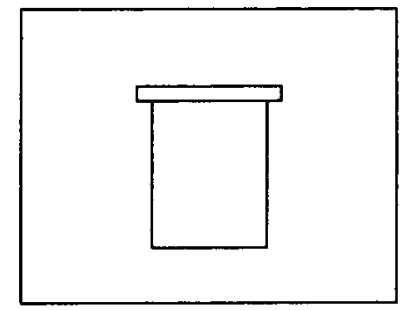

2. ADINTELADO CON ROSCA TRPEZOIDAL DE 0,30 Tipico del Siglo XMI

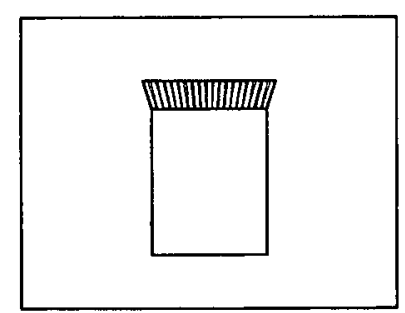

3. ADINTELADO CON ROSCA TRAPEZOIDAL DE 0.45 SS. $X M I-X M I$

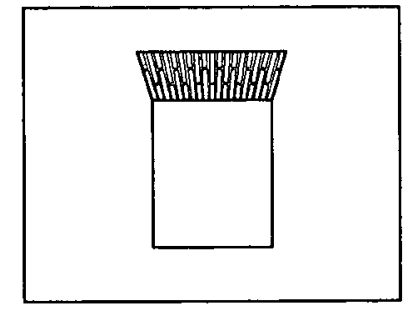

4. ADINTELADO CON ROSCA TRAPEZOIDAL MAYOR DE 0,45 Inicios del S. XM / Siglo XVII

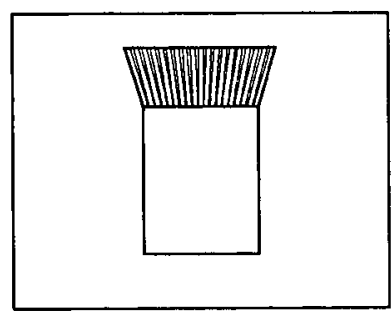

5. ADINTELADO CON ROSCA REBAJADA DE 0.30

Siglo XV. (Generalmente abocinado y modio punto rebajado en al reverso).

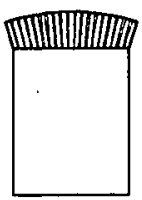

6. ABOCINAMIENTO CON ROSCA REBAJADA MAYOR DE 0,30 Y ABOCINAMIENTO CURVO EN EL REVERSO.

S5. $X V-X M I I$

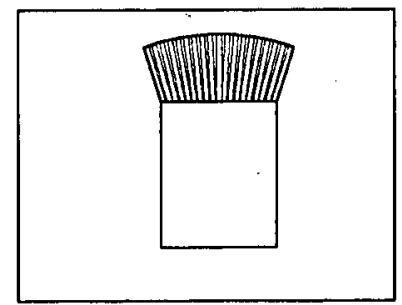

7. REBAJADO AL EXTERIOR Y ADINTELADO AL INTERIOR CON ROSCA TRAPEZOIDAL MAYOR DE 0,30.

is mitad del Siglo XIII

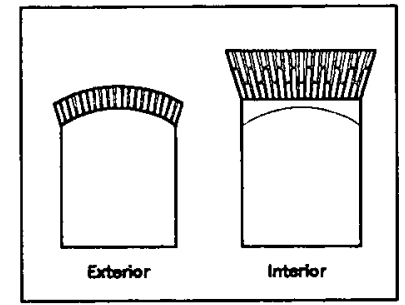

8. ADINTELADO TRAPEZOIDAL DE 0,45, ABOCINADO $Y$ RABAJADO AL INTERIOR CON ARCO DE DESACARGA REBAJADO DE 0.30 .

Ultimo cuarto del S. XV y primer cuarto del S. XM

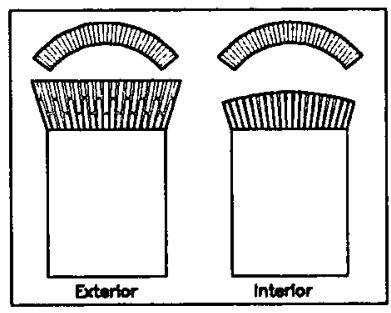

Fig. 12. Tipos de roscas y dinteles sevillanos. 1. (Tabales 1993) 
9. ADINTELADO SIN ORLA PERO CON DESCARGA DE MEDIO PUNTO REBALADA DE 0,30 .

Primer tercio del S. XMI

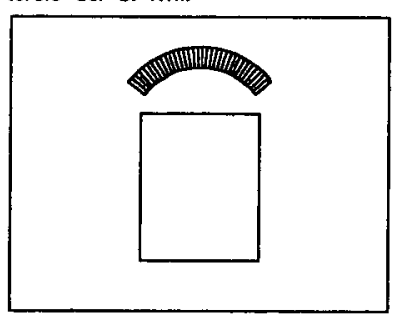

10. ADINTELADO ANCHO AL EXTERIOR Y ESCARZANO AL INTERIOR Y ABOCINADO.

Fines del S. XVI - Inicios del S. XMI.

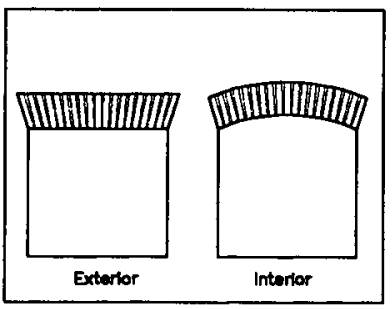

11. MEDIO PUNTO CON ROSCA DE 0,30 A AMBAS CARAS. Iniclos del S. XY - Inicios del S. XVI

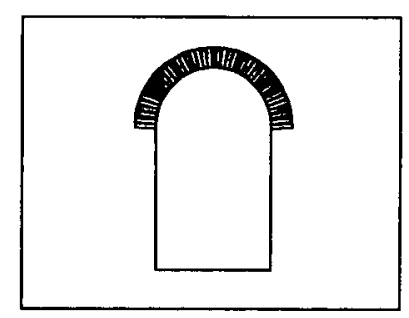

12. MEDIO PUNTO AL EXTERIOR Y ADINTELADO AL INTERIOR CON ROSCA DE 0,30.

Tercer tercio del S. XV

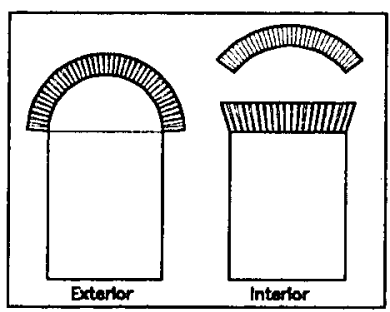

13. CARPANEL CON ROSCA DE UN PIE. Primera mitod del S. XVI

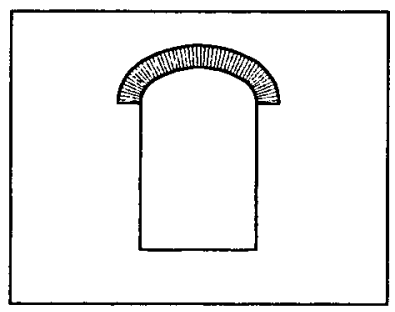

14. EACARZANO A AMBOS LADOS.

Siglo XM (mors en el primer cuorto).

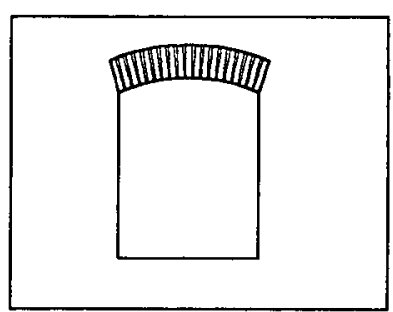

Fig. 13. Tipos de roscas y dinteles sevillanos. 1. (Tabales 1993) 


\section{TIPO DE ENLUCIDOS (S610 los m6́s comunes)}

1. ENLUCIDO DE CAL SIMPLE, FINO (grosor menor de 0,01).

2. ENLUCIDO DE CAL GRUESO (grosor mayor de 0,01).

3. ENLUCIDO DE CAL CON PINTURA MURAL.

4. ENJABELGADO.

5. AVITOLADO.

6. ESGRAFIADO O CASETONES.

7. ESGRAFIADO ENLADRILLADO.

8. ESGRAFIADO GEOMETRICO.

9. ESGRAFIADO FLORAL.

10. ESCAYOLA O YESO.

11. REPELLADO TOSCO.

12. ENCALADO.

13. ENFOSCADO DE CAL.

14. CEMENTO.

\section{TIPOS DE AÑADIDOS (Solo los mos comunes)}
1. METAL.
2. VIDRIO.
3. MADERA.
4. CERAMICA.
5. YESERIA.
6. AZULEJERIA.
7. CANERIAS.
8. LADRILLOS.
9. ELEMENTO PETREO.
10. BOVEDA.
11. HORMIGON.
12. CABLE / MATERIAL ELECTRICO.

Fig. 14. Clasificación simplificada de enlucidos y añadidos paramentales. (Tabales 1994) 


\section{BANOMEDIEVALES}

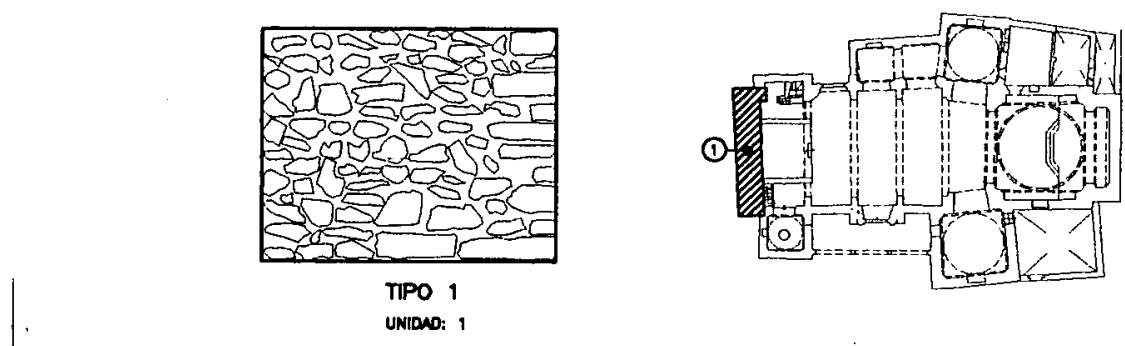

FINES DEL $S . X V$ - INICIOS DEL $S . X M$

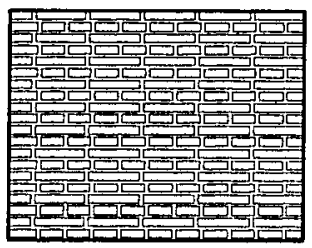

TIPO 1

UNDWDES: $\mathbf{8 , 8}$

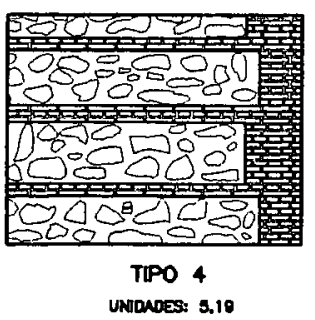

UNDNoEs: 5.10

MEDIADOS DEL S. XVII - SIGLO XVIII

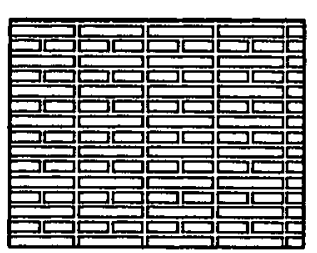

TIPO 1

UNIDND: 38

SS. $X \mathrm{X} \mid 1-\mathrm{XIX}$

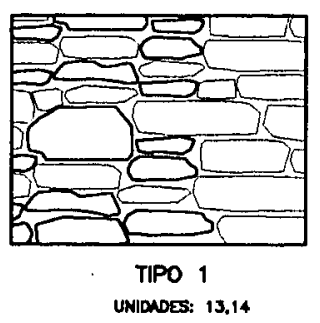

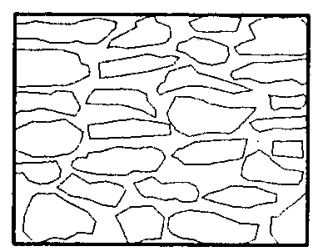

TIPO 2

UNIDUDES: $13,14,15,18,17$

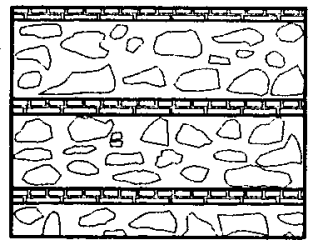

TIPO 5

UNADWDES: $7.8,9$

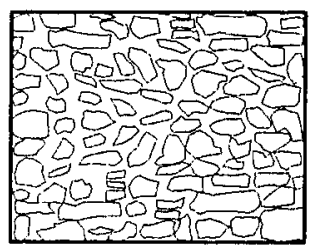

TIPO 3

UNDNOES: 2,3,4

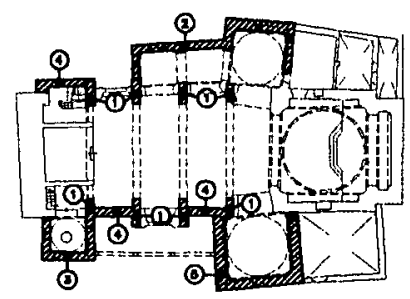

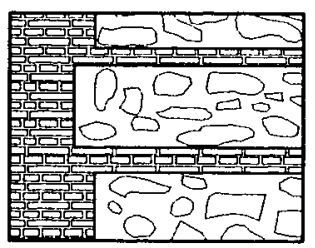

TIPO 2

UNIDNOES: $10,11,31$
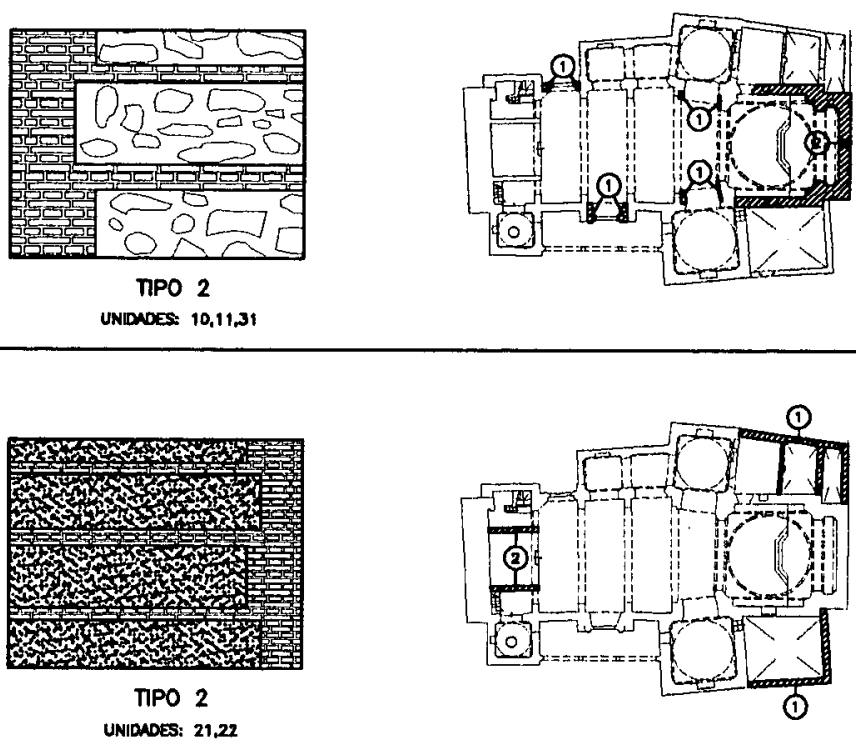

Fig. 15. Ejemplo de aplicación de la tabla de aparejos. Iglesia de Santa Ana, Guadalcanal, Sevilla. (Tabales 1996). 


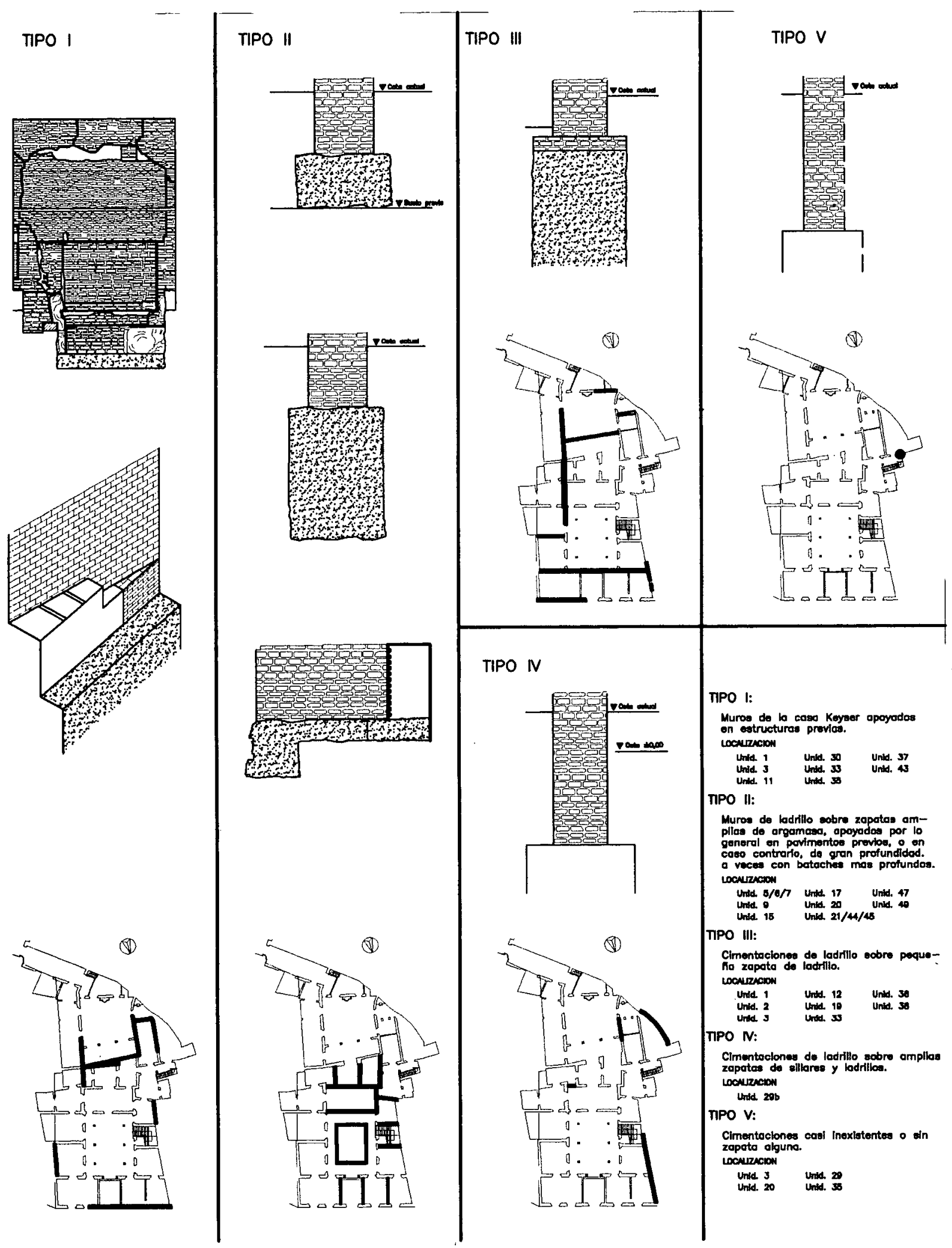

Fig. 16. Ejemplo de tipología de cimientos iniciada en el Palacio de Conde de Ibarra 18. Sevilla (Tabales, Pozo, Oliva, 1994). 
ARCOS GOTICOS MUDEJARES

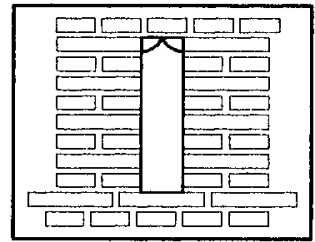

TIPO 1

SAETERA CONOPINL

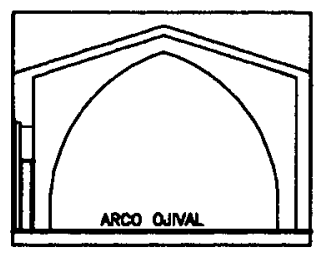

TPO 2

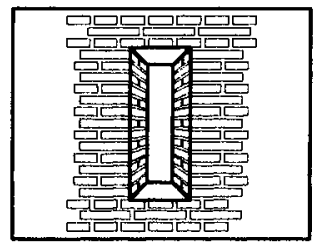

TIPO 3

SAETER ABOCIMUDA

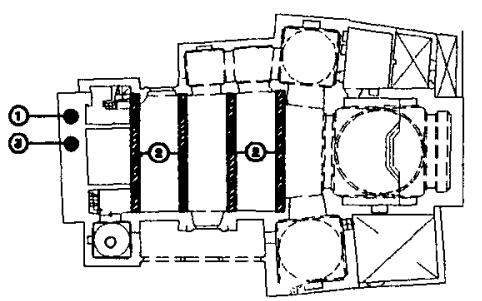

ARCOS DE INICIOS DEL S. XVI

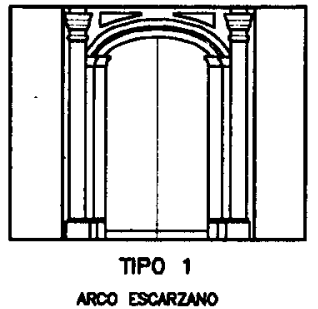

ARCO ESCARZNO

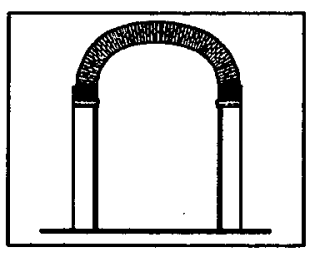

TIPO 2

ARCO CARPNEI

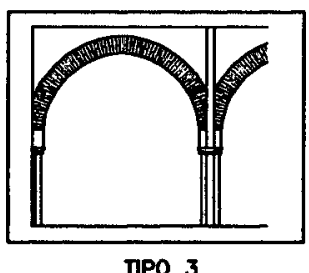

TIPO 3 MEDD PUNTO LOERAMERTE APUNTADO

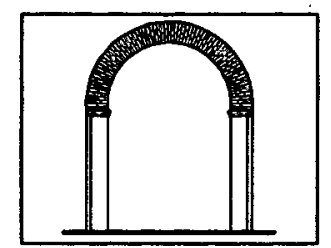

TIPO 4 MED 10 PUNTO

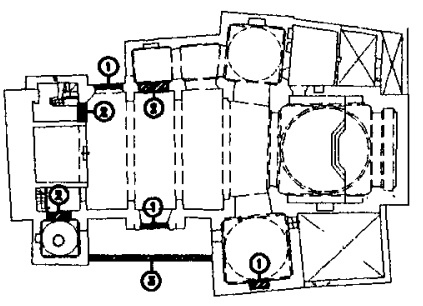

ARCOS Y DINTELES BARROCOS

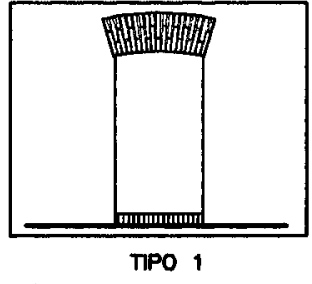

DINTE ABOCINADO

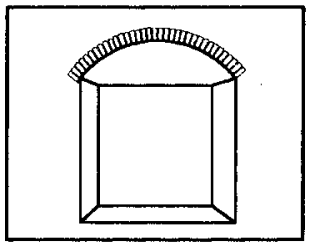

TIPO 2

ARCO NBOCANWDO Y RERANDO
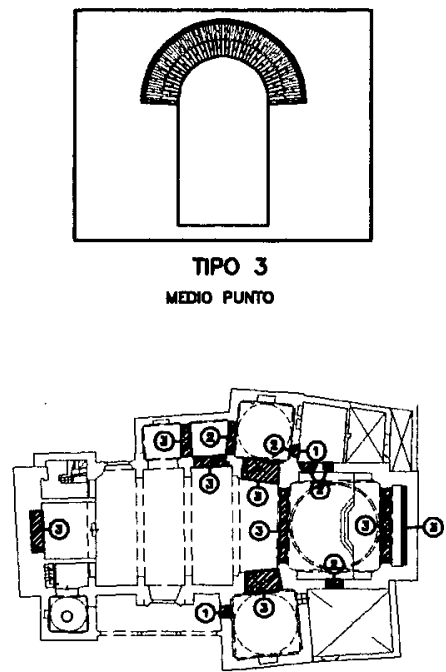

Fig.17. Ejemplo de aplicación de la tabla de vanos en Santa Ana. (Tabales 1996) 

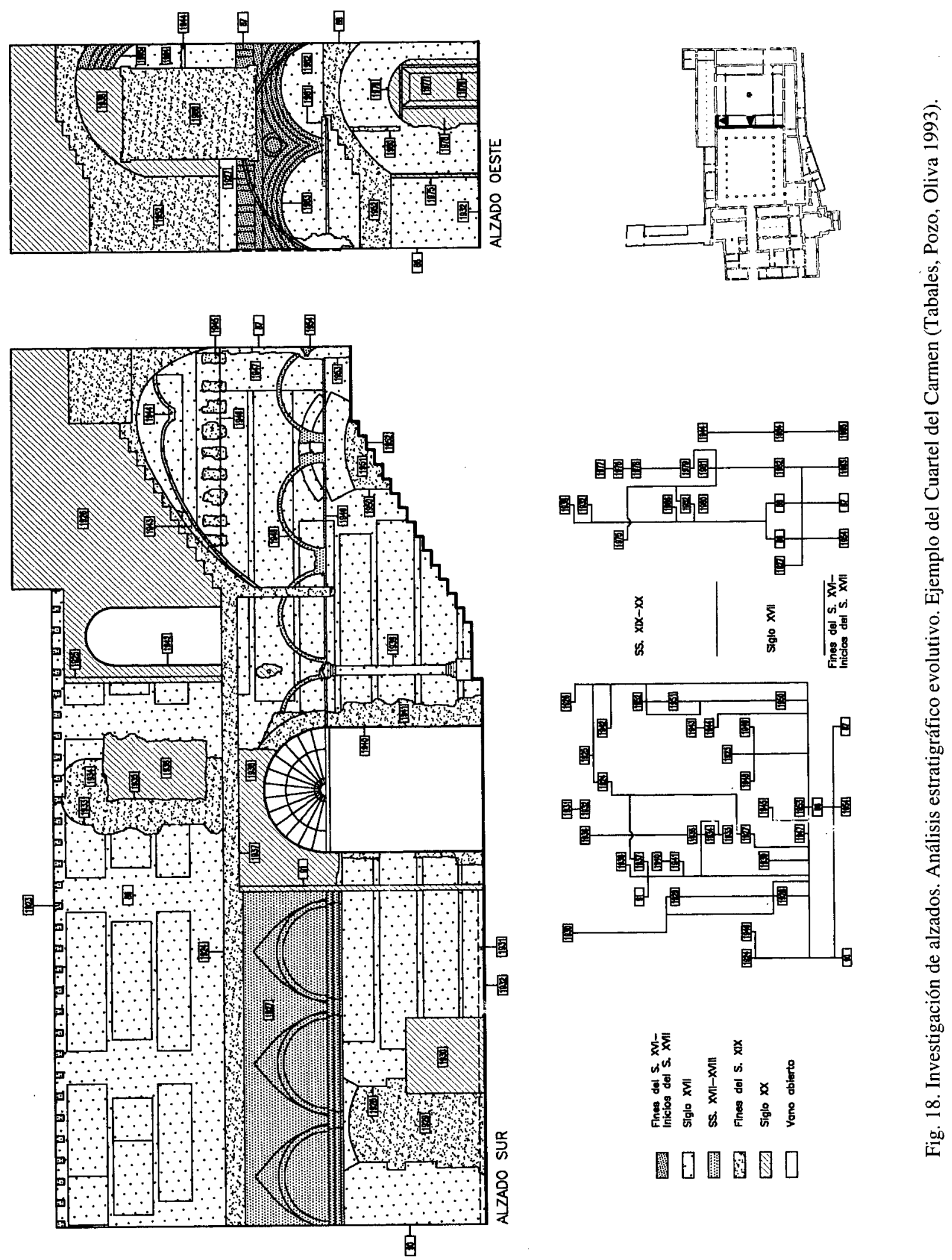

ISSN: 1133-4525 ISSN-e: 2255-3924 

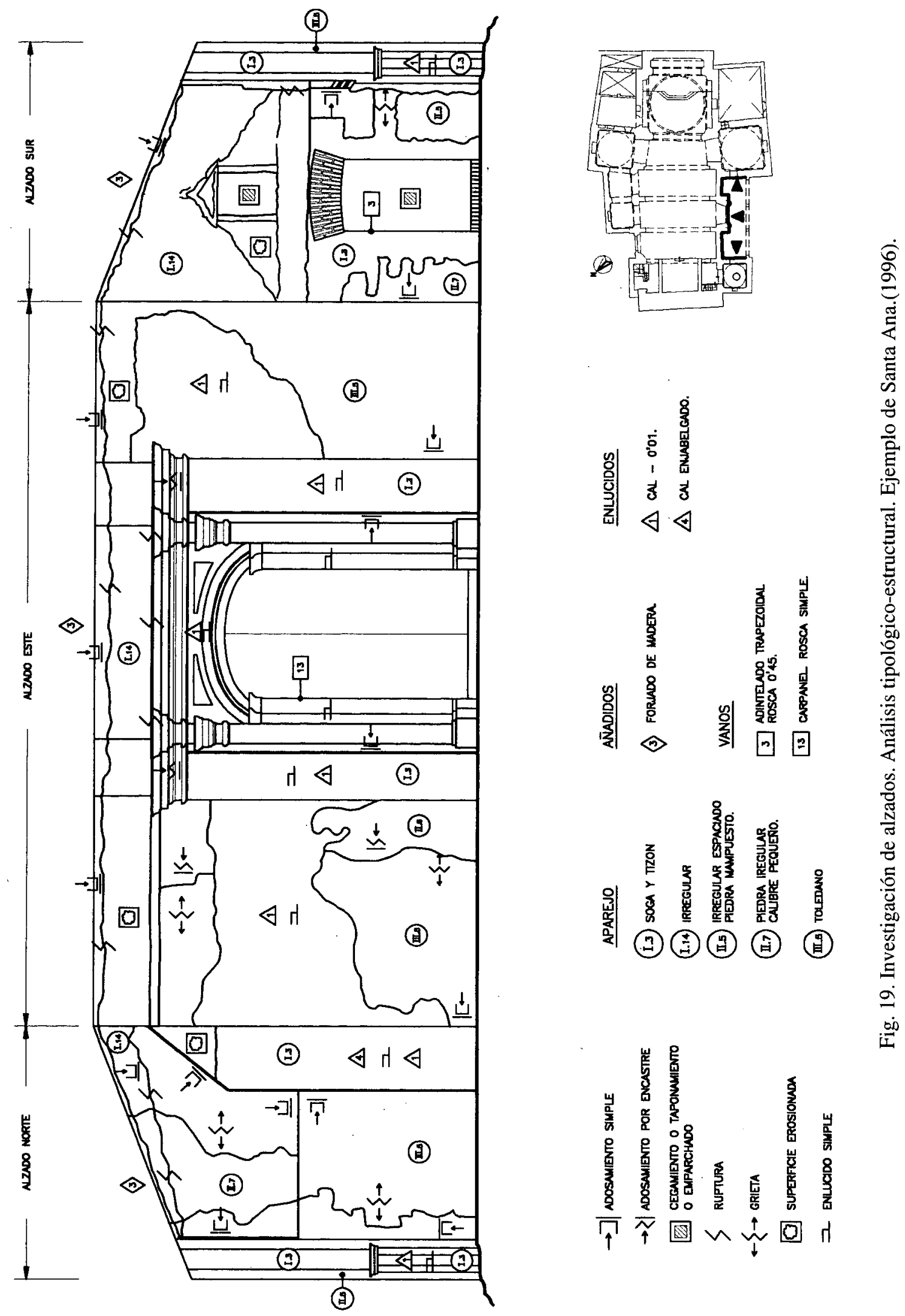


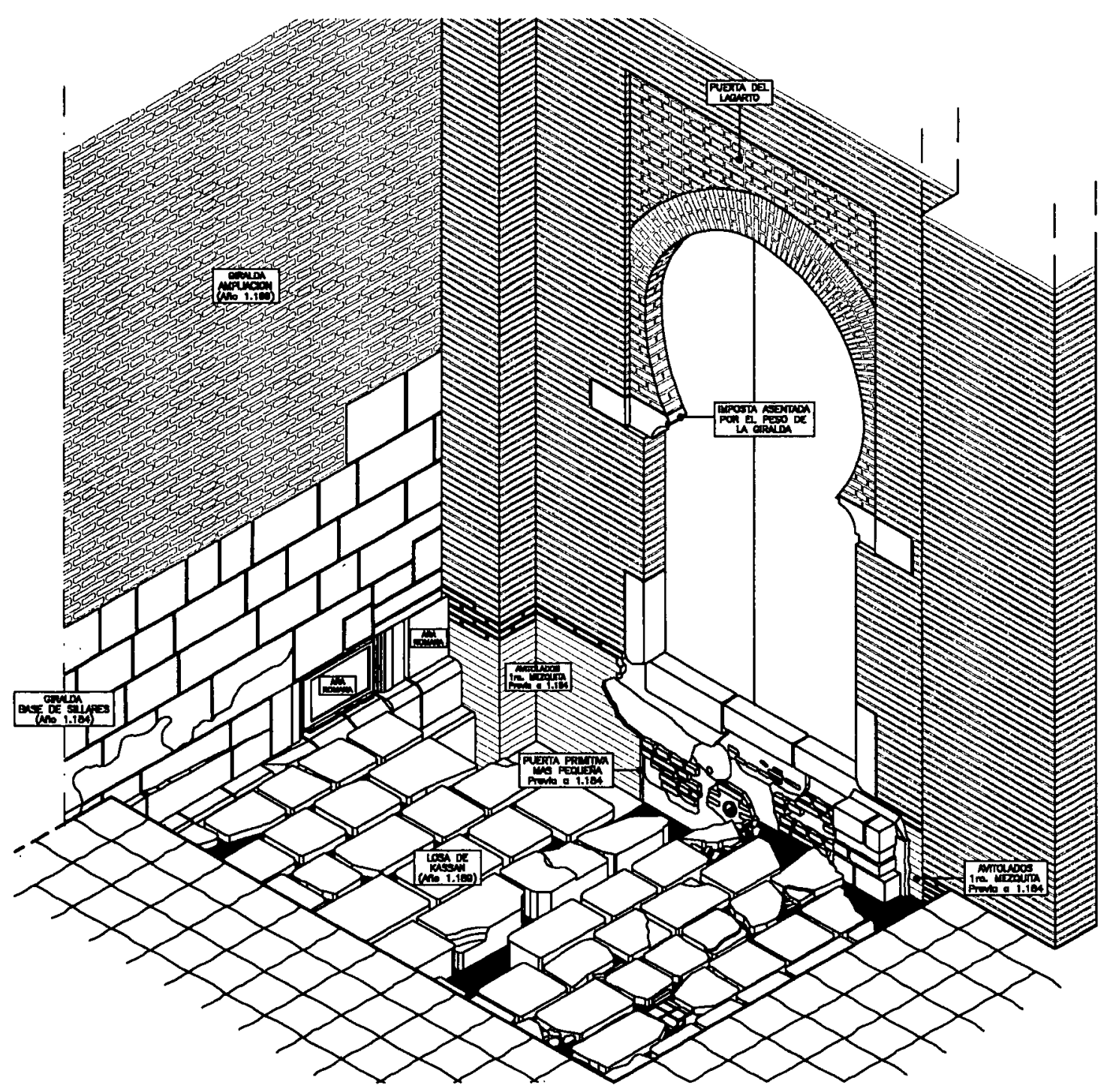

Fig.20. Catedral de Sevilla; Puerta del Lagarto y Giralda; identificación eventos principales. (Tabales 1996). 


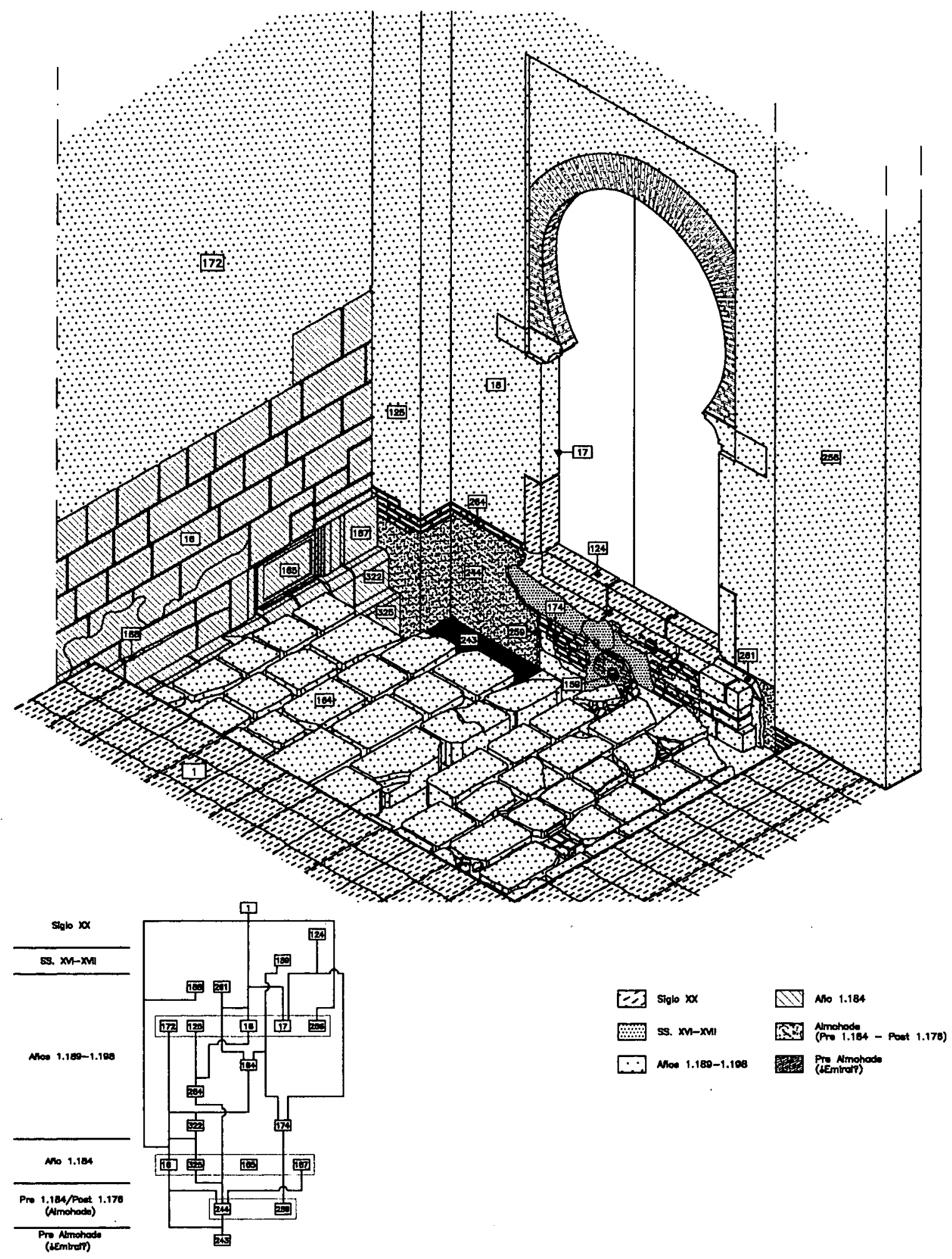

Fig. 21. Catedral de Sevilla; Puerta del Lagarto y Giralda; Análisis estratigráfico. 


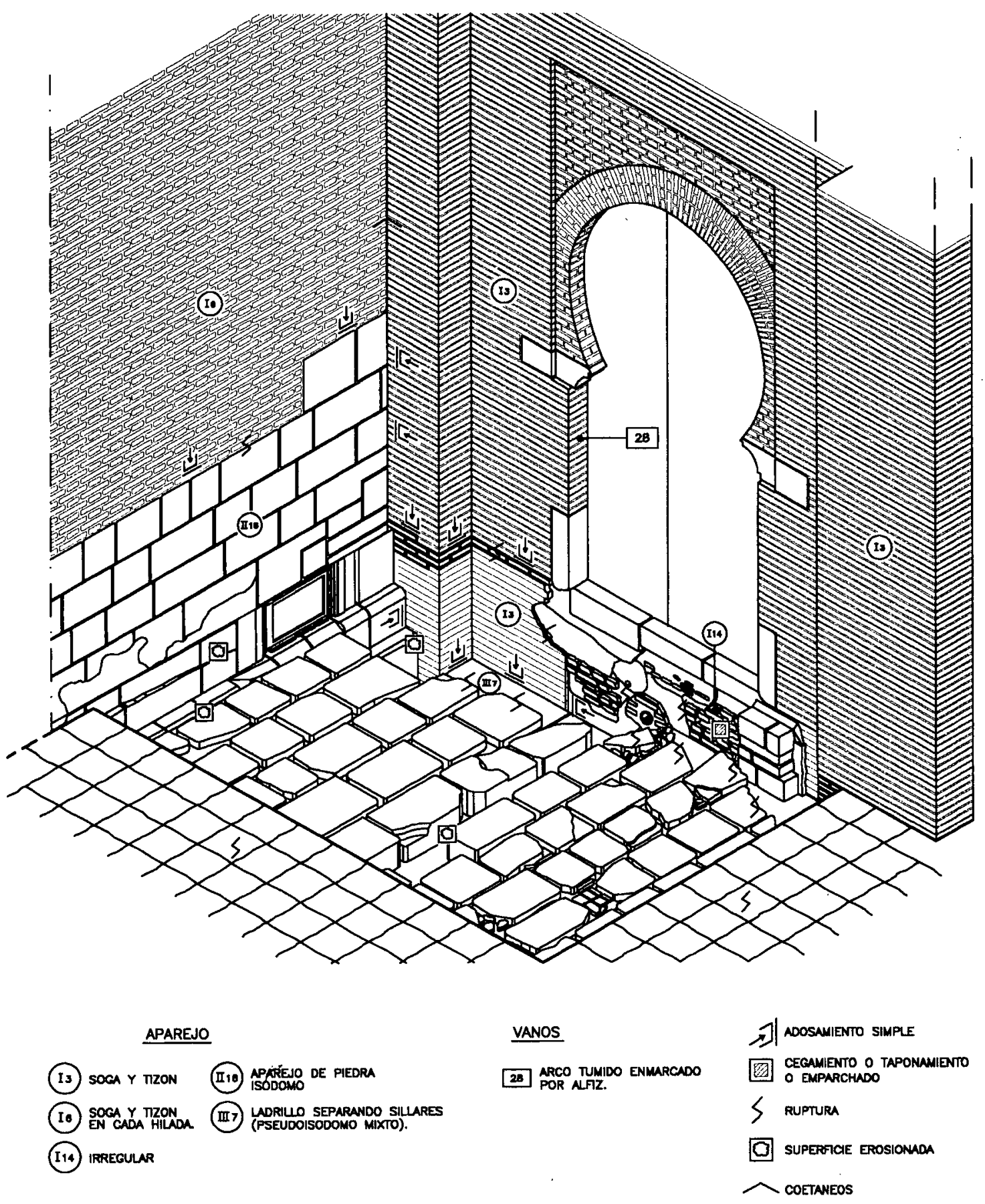

Fig. 22. Catedral de Sevilla; Puerta del Lagarto y Giralda; Análisis tipológico-estructural. 